\title{
A unified framework for three accelerated extragradient methods and further acceleration for variational inequality problems
}

D. R. Sahu ( $\nabla$ drsahudr@gmail.com )

Banaras Hindu University

\section{Research Article}

Keywords: Algorithmic operator, Seed operator, Pseudo-monotone operator, Extragradient method, Inertial Mann iterative method, Normal S-iterative method, Property (A ), Variational inequality problem

Posted Date: July 23rd, 2021

DOl: https://doi.org/10.21203/rs.3.rs-552362/v1

License: (1) This work is licensed under a Creative Commons Attribution 4.0 International License.

Read Full License 
Noname manuscript No.

(will be inserted by the editor)

\title{
A unified framework for three accelerated extragradient methods and further acceleration for variational inequality problems
}

\author{
D. R. Sahu \\ Received: date / Accepted: date
}

\begin{abstract}
The main strategy of this paper is intended to speed up the convergence of the inertial Mann iterative method and further speed up it through the normal S-iterative method for a certain class of nonexpansive type operators that are linked with variational inequality problems. Our new convergence theory permits us to settle down the difficulty of unification of Korpelevich's extragradient method, Tseng's extragardient method, and subgardient extragardient method for solving variational inequality problems through an auxiliary algorithmic operator, which is associated with seed operator. The paper establishes an interesting fact that the relaxed inertial normal S-iterative extragradient methods do influence much more on convergence behaviour. Finally, the numerical experiments are carried out to illustrate that the relaxed inertial iterative methods, in particular the relaxed inertial normal S-iterative extragradient methods, may have a number of advantages over other methods in computing solutions of variational inequality problems in many cases.
\end{abstract}

Keywords Algorithmic operator · Seed operator · Pseudo-monotone operator · Extragradient method $\cdot$ Inertial Mann iterative method $\cdot$ Normal S-iterative method · Property $(\mathscr{A}) \cdot$ Variational inequality problem

Mathematics Subject Classification (2000) 47H05 - 47J26 · 47N10

\section{Introduction}

1.1 Convergence rates of iterative methods for nonexpansive operators

Many problems arising in different areas of mathematics such as signal/image recovery, optimization, variational analysis, differential and integral equations, etc. can be modeled by the fixed point problem (FPP):

$$
\text { to find } x \in C \text { such that } x=S x \text {, }
$$

where $S: X \rightarrow C$ is an operator and $C$ is a nonempty subset of a Hilbert space $X$. The solutions to this problem are called fixed points of the operator $S$. We denote the set of solutions of $\mathbf{F P P}(1)$ (or equivalently set of fixed points of $S$ ) by

$$
\operatorname{Fix}(S)=\{x \in C: x=S x\} .
$$

There is a vast literature on the topic "computation of fixed points" relative to important aspects such as: types of involved operators, robustness, efficiency and convergence rate. It is well

D. R. Sahu

Department of Mathematics, Institute of Science, Banaras Hindu University, Varanasi-221005, India

E-mail: drsahudr@gmail.com

Orcid=0000-0003-0994-4529 
known that the Picard iterative method for nonexpansive operators may, in general, not behave well (see $[1,11,38])$. One of the powerful iterative techniques for computation of fixed points of nonexpansive-type operators in Hilbert spaces as well as Banach spaces is Mann iterative method, which was introduced by Mann [23] in 1953 as follows:

$$
x_{n+1}=\left(1-\alpha_{n}\right) x_{n}+\alpha_{n} T x_{n} \text { for all } n \in \mathbb{N},
$$

where $\left\{\alpha_{n}\right\}$ is real sequence in $[0,1]$ and $T: C \rightarrow C$ is nonexpansive on closed convex set $C$ of suitable Banach space. This method has been extensively studied and applied by many authors in various science and engineering problems, see for instance $[1,5,7]$.

The augmentation of rates of convergence of iterative techniques fascinate to researchers theoretically as well as numerically for various nonlinear problems. In order to accelerate the convergence rate, multi-step iterative algorithms have been studied by using additional inertial terms (see $[8,12,13,22,24,26,36]$ ). The inertial term is motivated from heavy ball method proposed by Polyak [24]. The heavy ball method is a discretization of second order ordinary differential equation

$$
x^{\prime \prime}(t)+\gamma x^{\prime}(t)+\nabla \omega(x(t))=0,
$$

where $x(t)$ is a time continuous trajectory and $\gamma>0$ is a friction and $\omega(x(t))$ is an external gravitational field. In particular, Mainge [22] adopted the inertial Mann iterative method for computation of fixed points of a nonexpansive operator $T$ defined in a Hilbert space $X$ as follows:

$$
\left\{\begin{array}{l}
y_{n}=x_{n}+\theta_{n}\left(x_{n}-x_{n-1}\right), \\
x_{n+1}=\left(1-\alpha_{n}\right) y_{n}+\alpha_{n} T y_{n} \text { for all } n \in \mathbb{N} .
\end{array}\right.
$$

Mainge [22] studied weak convergence of algorithm (2) under the following conditions:

(M0) $\theta_{n} \in[0, \theta]$ for all $n \in \mathbb{N}$ and for some $\theta \in[0,1)$;

(M1) $\sum_{n=1}^{\infty} \theta_{n}\left\|x_{n}-x_{n-1}\right\|^{2}<\infty$.

The verification of condition $(M 1)$ is laborious in practical situations. Bot and Csetneck [8] proved a result for computation of fixed points of nonexpansive operators under some different condition without the condition (M1). Recently, author [26] studied weak convergence and convergence rate of inertial Mann iterative method (2) for computation of fixed points of quasinonexpansive operator $T$ with affine domain in a Hilbert space under the practical condition:

$$
0<a \leq \alpha_{n} \leq b<1 \text { for all } n \in \mathbb{N},
$$

which is more simpler than the condition (6) of Bot and Csetneck [8, Theorem 5].

\subsection{Variational inequality problems}

It is well known that Tseng's extragradient method and subgardient extragardient method, which require in every step only one projection operation on $C$, are valuable alternatives for Korpelevich's extragradient method for solving variational inequality problems over a convex closed set $C$ governed by monotone/ pseudo-monotone and Lipschitz continuous operators.

We now consider the following nonlinear problem:

$$
\text { find } x^{*} \in C \text { such that }\left\langle F x^{*}, x-x^{*}\right\rangle \geq 0 \text { for all } x \in C \text {, }
$$

where $C$ is a nonempty closed convex subset of a real Hilbert space $X$ and $F: C \rightarrow X$ is a nonlinear operator. The problem (3) is called variational inequality problem over $C$ for the operator $F: C \rightarrow X$ and it is denoted by $\operatorname{VI}(C, F)$. The solution set of variational inequality problem $\mathrm{VI}(C, F)$ is denoted by $\Omega[\mathrm{VI}(C, F)]$.

Many problems in convex optimization, economics, engineering mechanics and mathematical physics can be Modeled as variational inequality problems (3). It is well known that the convergence of projected gradient iterative method introduced by Goldstein [16] requires the strong 
monotonicity of the operator $F$ involved in (3). Without strong monotonicity of the operator $F$, the sequence generated by projection method may diverge. In order to relax strong monotonicity of the operator $F$, Korpelevich [20] introduced an extragradient method (KEM) for solving variational inequality problem $\mathrm{VI}(C, F)$ as follows:

$$
x_{n+1}=P_{C}\left(x_{n}-\lambda F P_{C}\left(x_{n}-\lambda F x_{n}\right)\right) \text { for all } n \in \mathbb{N},
$$

where $F: C \rightarrow X$ is a monotone and $L$-Lipschitz continuous operator and $\lambda \in(0,1 / L)$.

Korpelevich's algorithm and its variants have been studied by many authors, see for instance, $[6,39]$. In subsequent work, Dong et al. [14] introduced an extragradient method with inertial effect (IM-KEM) by combining the extragradient algorithm ( [20]) and inertial extrapolation method ( [8]) for the solution of variational inequality problem. The inertial extragradient method in [14] is given as follows:

$$
\left\{\begin{array}{l}
w_{n}=x_{n}+\theta_{n}\left(x_{n}-x_{n-1}\right) \\
y_{n}=P_{C}\left(w_{n}-\tau F w_{n}\right) \\
x_{n+1}=\left(1-\alpha_{n}\right) w_{n}+\alpha_{n} P_{C}\left(w_{n}-\tau F y_{n}\right) \text { for all } n \in \mathbb{N}
\end{array}\right.
$$

where $F: C \rightarrow X$ is a monotone and $L$-Lipschitz continuous operator and $\left\{\theta_{n}\right\}$ is nondecreasing with $\theta_{1}=0$ and $0 \leq \theta_{n}<\theta<1$ for all $n \in \mathbb{N}$ and $\lambda, \sigma, \delta>0$ such that $\delta>\frac{\theta \xi}{1-\tau^{2} L^{2}}$ and

$$
0<\alpha \leq \alpha_{n} \leq \frac{\delta\left(1-\tau^{2} L^{2}\right)-\theta \xi}{\delta \xi},
$$

where $\xi=(1+\tau L)^{2} \theta(1+\theta)+\left(1-\tau^{2} L^{2}\right) \theta \sigma+\sigma(1+\tau L)^{2}$.

On the other hand, Tseng [35] introduced the following extragradient method (TEM) for solving variational inequality problem (3):

$$
x_{n+1}=P_{C}\left(x_{n}-\lambda F x_{n}\right)-\lambda\left(F P_{C}\left(x-\lambda F x_{n}\right)-F x_{n}\right) \text { for all } n \in \mathbb{N},
$$

where $\lambda \in(0,1 / L)$. Recently, the extragradient methods have received great attention for solving pseudo-monotone variational inequality problems in infinite dimensional Hilbert spaces $[9,19,21$, 37]. Censor et al. [10] proposed the following subgradient extragradient method (SEM) for solving variational inequality problem (3) (3):

$$
\left\{\begin{array}{l}
y_{n}=P_{C}\left(x_{n}-\lambda F x_{n}\right) \\
x_{n+1}=P_{H_{n}}\left(x_{n}-\lambda F x_{n}\right) \text { for all } n \in \mathbb{N}
\end{array}\right.
$$

where $H_{n}=\left\{w \in X:\left\langle x_{n}-\lambda F x_{n}-y_{n}, w-y_{n}\right\rangle \leq 0\right\}$. In [9], Bot et. al studied a relaxed extragradient method and compared that the performance of relaxed extragradient method better than subgradient-extragradient method (8). Indeed, the comparison of inertial version of the relaxed extragradient method and that of relaxed subgradient-extragradient method are not studied in Bot et. al [9]. Moreover, other interesting observations are given in the next subsection.

\subsection{Observations}

The following are remarks are given in [10]:

(R1) If projections onto $C$ are easily executed, then extragradient method (4) is particularly useful.

(R2) If $C$ is a general closed and convex set, then a minimal distance problem has to be solved (twice) in order to obtain the next iterate. This might seriously affect the efficiency of the extragradient method (4).

In view of remarks (R1) and (R1), there are the following natural questions:

(Q1) Is it possible to unify $\operatorname{KEM}(4), \operatorname{TEM}(7)$ and $\operatorname{SEM}(8)$ for pseudo-monotone variational inequality problems by defining a certain class of algorithmic operators? 
(Q2) If projection onto $C$ is executed, how to compare convergence rates of $\operatorname{KEM}(4), \operatorname{TEM}(7)$ and $\operatorname{SEM}(8) ?$

In the light of Dong et al. [14], we have the following observations:

(O1) The upper bound of the iteration parameter $\left\{\alpha_{n}\right\}$ determined by (6) depends on other parameters $\tau, \sigma, \delta, \theta$ and $L$. Therefore, the determination of upper bound of $\left\{\alpha_{n}\right\}$ for convergence behaviour of the extragradient method (5) is not easy.

(O2) The rate of convergence of inertial Mann iterative method (5) is not reported in [14].

(O3) There are a huge number of variants of extragradient methods for the computational solutions of variational inequality problems involving monotone/pseudo-monotone operators.

\subsection{Contributions}

Motivated by questions (Q1)-(Q2) and observations $(\mathbf{O 1})-(\mathbf{O 3})$, the main objective of this paper is to unify characterizations of methodologies of extragradient methods $\mathrm{KEM}(4), \operatorname{TEM}(7)$ and $\operatorname{SEM}(8)$ for solving variational inequality problems involving monotone/pseudo-monotone operators. While doing so, first we investigate convergence theory of two broad classes of the relaxed inertial iterative methods generated by strongly quasi-nonexpansive operators in Hilbert spaces for computation of solutions of $\mathbf{F P P}(1)$ under the practical assumptions (see Section 3). Our convergence theory strengthens the situation that if projection onto $C$ is executed, then Korpelevich's extragradient method (4) behaves well like Tseng's extragardient method (7) and subgardient extragardient method (8). Our convergence theory provides affirmative answers of questions (Q1)-(Q2).

\subsection{Organization of the paper}

This paper is organized as follows: In Section 2, we summarize some basic definitions and results. In Section 3, first we introduce the relaxed inertial iterative method based on Mann iteration method and to further speed up the relaxed inertial Mann iterative method, we introduce normal S-iteration method with the relaxed iteration parameters $\left\{\alpha_{n}\right\}$ in $(0, \infty)$ and establish their convergence theory with convergence rate in worst case for computation of solutions of $\mathbf{F P P}(1)$ when there exists an strongly quasi-nonexpansive operator $T: X \rightarrow X$, which is associated with original operator $S$. Indeed, the rate of convergence of the relaxed inertial Mann iterative method is estimated as $R_{T,\left\{w_{n}\right\}}(n)=o\left(\frac{1}{\sqrt{n}}\right)$ whereas the rate of convergence of the relaxed inertial normal S-iterative method is estimated as $R_{T,\left\{x_{n}\right\}}(n)=o\left(\frac{1}{\sqrt{n}}\right)$. In Section 4.1, we combine the relaxed inertial Mann iterative method and the relaxed inertial normal S-iterative method with three basic extragradient methods (4), (7) and (8) and develop corresponding relaxed inertial extragradient methods for solving pseudo-monotone variational inequality problems. In Section 5 , we give numerical test problems for demonstrating the convergence behaviour of six new developed extragradient methods. Finally, in the last section, we conclude the paper with some future plan of research work.

\section{Mathematical preliminaries}

Throughout the paper, $X$ denotes a real Hilbert space with inner product $\langle\cdot, \cdot\rangle$ and norm $\|\cdot\|$, respectively. The strong (weak) convergence of a sequence $\left\{x_{n}\right\}$ to $x$ is denoted by $x_{n} \rightarrow x$ $\left(x_{n} \rightarrow x\right)$, respectively. Let $\left\{x_{n}\right\}$ be a sequence in $X, C$ a nonempty subset of $X$ and $U: X \rightarrow C$ an operator. Define $r_{U,\left\{x_{n}\right\}}(n)=\min \left\{\left\|x_{i}-U x_{i}\right\|: i=1,2, \ldots, n\right\}$ for all $n \in \mathbb{N}$.

One can see that $r_{U}(n):=r_{U,\left\{x_{n}\right\}}(n) \leq\left\|x_{i}-U x_{i}\right\|$ for all $i=1,2, \ldots, n$ and the sequence $\left\{r_{U}(n)\right\}_{n=1}^{\infty}$ is decreasing.

Let $C$ be a nonempty subset of $X$ and $T: C \rightarrow X$ an operator. $T$ is said to be 
(i) nonexpansive if $\|T x-T y\| \leq\|x-y\|$ for all $x, y \in C$;

(ii) firmly nonexpansive if $\|T x-T y\|^{2} \leq\|x-y\|^{2}-\|x-y-(T x-T y)\|^{2}$ for all $x, y \in C$;

(iii) quasi-nonexpansive if $\operatorname{Fix}(T) \neq \emptyset$ and $\|T x-y\| \leq\|x-y\|$ for all $x \in C$ and $y \in \operatorname{Fix}(T)$;

(iv) $\kappa$-strongly quasi-nonexpansive if $\operatorname{Fix}(T) \neq \emptyset$ and there exists a constant $\kappa \in(0, \infty)$ such that

$$
\|T x-y\|^{2} \leq\|x-y\|^{2}-\kappa\|x-T x\|^{2} \text { for all } x \in C \text { and } y \in \operatorname{Fix}(T) ;
$$

(v) $L$-Lipschitz continuous if there exists a constant $L \in(0, \infty)$ such that $\|T x-T y\| \leq L\|x-y\|$ for all $x, y \in C$;

(vi) bounded if $D$ is bounded in $C$ implies $T(D)$ is bounded.

An operator $F: C \rightarrow X$ is said to be

(i) monotone if $\langle F x-F y, x-y\rangle \geq 0$ for all $x, y \in C$,

(ii) pseudo-monotone if $\langle F x, y-x\rangle \geq 0 \Rightarrow\langle F y, y-x\rangle \geq 0$ for all $x, y \in C$.

A monotone operator $A$ is said to be maximal monotone if there is no proper monotone extension of $A$.

Definition 2.1 (Kato [18]) Let $C$ be a nonempty subset of a $X$ and $F: C \rightarrow X$ an operator. Then $F$ is said to be hemi-continuous at $x \in C$ if for any $y \in X$ and $t>0$ such that $x+t y \in C$, we have $F(x+t y) \rightarrow F x$ as $t \rightarrow 0 . F$ is hemi-continuous on $C$ if it is hemi-continuous at each point of $C$.

Remark 2.1 If $f: X \rightarrow \mathbb{R}$ is a continuously Fréchet differentiable convex function, then the gradient $\nabla f$ of $f$ is hemi-continuous maximal monotone operator.

Let $C$ be a nonempty closed convex subset of $X$. For every element $x \in X$, there exists a unique nearest point in $C$, denoted by $P_{C} x$, such that

$$
\left\|x-P_{C} x\right\|=\inf \{\|x-y\|: y \in C\} .
$$

The operator $P_{C}: X \rightarrow C$ is called the projection operator onto $C$.

Lemma 2.1 (see [1]) Let $C$ be a nonempty closed convex subset of $X$ and $P_{C}$ be the metric projection from $X$ onto $C$. Then the following hold:

(a) $\left\langle x-P_{C} x, y-P_{C} x\right\rangle \leq 0$ for all $x \in X$ and $y \in C$;

(b) $\left\|P_{C} x-P_{C} y\right\| \leq\|x-y\|$ for all $x, y \in X$;

(c) $\left\|P_{C} x-P_{C} y\right\|^{2} \leq\left\langle x-y, P_{C} x-P_{C} y\right\rangle$ for all $x, y \in X$;

(d) $\left\|P_{C} x-y\right\|^{2} \leq\|x-y\|^{2}-\left\|\left(I-P_{C}\right) x\right\|^{2}$ for all $x \in X$ and $y \in C$.

The property $(\mathscr{A})$ plays an important role for finding fixed points (common fixed points) of nonexpansive (family of nonexpansive operators) and zero of monotone operators (see [27,29,30]). Following [29], we define the property $(\mathscr{A})$ of an operator $S: X \rightarrow C$ with respect to the operator $T: X \rightarrow X$ when $C$ is a nonempty subset of $X$.

Definition 2.2 Let $C$ be a nonempty subset of $X$ and let $S: X \rightarrow C$ and $T: X \rightarrow X$ be operators. We say that the operator $S$ has the property $(\mathscr{A})$ with respect to the operator $T$ if the following holds:

for any bounded sequence $\left\{x_{n}\right\}$ in $X$, we have

$$
\lim _{n \rightarrow \infty}\left\|x_{n}-T x_{n}\right\|=0 \Rightarrow \lim _{n \rightarrow \infty}\left\|x_{n}-S x_{n}\right\|=0 .
$$

Definition 2.3 Let $C$ be a nonempty subset of $X$ and $S: X \rightarrow C$ an operator. Then $I-S$ is said to be demiclosed at zero if $\left\{x_{n}\right\}$ is a sequence in $X$ such that $x_{n} \rightarrow x$ for some $x \in X$ and $x_{n}-S x_{n} \rightarrow 0$, we have $x-S x=0$.

Following Sahu [26, Proposition 2.2], we have 
Proposition 2.1 Let $C$ be a nonempty weakly closed subset of $X$ and $S: X \rightarrow C$ an operator such that $\operatorname{Fix}(S) \neq \emptyset$ and $I-S$ is demiclosed at zero. Let $\left\{x_{n}\right\}$ be a sequence in $X$ such that $\lim _{n \rightarrow \infty}\left\|x_{n}-v\right\|$ exists for all $v \in \operatorname{Fix}(S)$ and $\lim _{n \rightarrow \infty}\left\|x_{n}-S x_{n}\right\|=0$. If $\left\{x_{n}\right\}$ has a weakly convergence subsequence, then $\left\{x_{n}\right\}$ converges weakly to a fixed point of $S$.

Lemma 2.2 (see [7]) The following identities hold:

(i) $\|x-y\|^{2}=\|x\|^{2}+\|y\|^{2}-2\langle x, y\rangle$ for all $x, y \in X$,

(ii) $\|t x+(1-t) y\|^{2}=t\|x\|^{2}+(1-t)\|y\|^{2}-t(1-t)\|x-y\|^{2} \quad$ for all $t \in \mathbb{R}$ and $x, y \in X$.

Let $x, y \in X, \alpha \geq 0$ and $\rho>0$. Following Sahu [26], we have

$$
\|x+y\|^{2} \geq(1-\rho)\|x\|^{2}+\left(1-\frac{1}{\rho}\right)\|y\|^{2}
$$

and

$$
\|x-\alpha y\|^{2} \geq(1-\alpha)\|x\|^{2}-\alpha(1-\alpha)\|y\|^{2} .
$$

Lemma 2.3 (see [15]) Let $\left\{\beta_{n}\right\}$ and $\left\{\gamma_{n}\right\}$ be sequences of positive numbers such that $\sum_{n=1}^{\infty} \beta_{n} \gamma_{n}<$ $\infty$. Suppose that the sequence $\left\{\beta_{n}\right\}$ is nonsummable and the sequence $\left\{\gamma_{n}\right\}$ is decreasing. Then $\gamma_{n}=o\left(\frac{1}{\sum_{k=1}^{n} \beta_{k}}\right)$, where the notation o means that $s_{n}=o\left(1 / t_{n}\right)$ if and only if $\lim _{n \rightarrow \infty} s_{n} t_{n}=0$.

Lemma 2.4 (see [3]) Let $\left\{a_{n}\right\},\left\{b_{n}\right\}$ and $\left\{\theta_{n}\right\}$ be a sequence in $[0, \infty)$ such that

$$
a_{n+1} \leq a_{n}+\theta_{n}\left(a_{n}-a_{n-1}\right)+b_{n} \text { for all } n \in \mathbb{N},
$$

$\sum_{n=1}^{\infty} b_{n}<\infty$ and there exists a real number $\theta$ with $0 \leq \theta_{n} \leq \theta<1$ for all $n \in \mathbb{N}$. Then the following hold:

(i) $\sum_{n=1}^{\infty}\left[a_{n}-a_{n-1}\right]_{+}<\infty$, where $[t]_{+}=\max \{t, 0\}$,

(ii) there exists $a^{*} \in[0, \infty)$ such that $a_{n} \rightarrow a^{*}$.

Lemma 2.5 Let $C$ be a nonempty subset of $X, v \in C$ and $\left\{x_{n}\right\}_{n \geq 0}$ a sequence in $X$ satisfying the inequality:

$$
\begin{aligned}
\left\|x_{n+1}-v\right\|^{2} \leq & \left(1+\theta_{n}\right)\left\|x_{n}-v\right\|^{2}-\theta_{n}\left\|x_{n-1}-v\right\|^{2}-\varepsilon\left(1-\theta_{n}\right)\left\|x_{n+1}-x_{n}\right\|^{2} \\
& +\theta_{n}\left(1+\theta_{n}+\varepsilon\left(1-\theta_{n}\right)\right)\left\|x_{n}-x_{n-1}\right\|^{2} \text { for all } n \in \mathbb{N},
\end{aligned}
$$

where $\varepsilon \in(0, \infty)$ and $\left\{\theta_{n}\right\}$ is an increasing sequence in $[0,1)$. Define

$$
\varphi_{n}=\left\|x_{n}-v\right\|^{2}-\theta_{n}\left\|x_{n-1}-v\right\|^{2}+\theta_{n}\left(1+\theta_{n}+\varepsilon\left(1-\theta_{n}\right)\right)\left\|x_{n}-x_{n-1}\right\|^{2} \text { for all } n \in \mathbb{N} .
$$

Assume that $\varphi_{1}>0$ and there exists $\theta \in(0,1)$ such that

$$
\theta_{n} \leq \theta<\delta(\varepsilon)=: \frac{\varepsilon}{1+\varepsilon+\max \{1, \varepsilon\}} \text { for all } n \in \mathbb{N} .
$$

Then, we have the following:

(a) $\left\{\varphi_{n}\right\}$ is non-increasing.

(b) $\sum_{n=1}^{\infty}\left\|x_{n+1}-x_{n}\right\|^{2}<\infty$.

(c) $\lim _{n \rightarrow \infty}\left\|x_{n}-v\right\|$ exists

Remark 2.2 The assumption " $\varphi_{1}>0$ " automatically holds if $x_{0}=x_{1}$ or $\theta_{1}=0$. 


\section{Accelerated computation methods for FPP (1) and their convergence analysis}

Motivated by the idea adopted in Sahu at el. [29], in this section, our main goal is to introduce and study convergence analysis of the relaxed inertial iterative methods based on Mann iteration method and normal S-iteration method, which involve algorithmic operator $T$ for solving $\mathbf{F P P}(1)$. First, we introduce the notion of algorithmic operator for computation of solutions of $\mathbf{F P P}(1)$.

Definition 3.1 Let $C$ be a nonempty subset of $X$ and let $S: X \rightarrow C$ be a given operator. Then an operator $T: X \rightarrow X$ is said to be an algorithmic operator for $\mathbf{F P P}(1)$ if the condition $(\mathbf{A} 0)$ holds:

(A0) $\operatorname{Fix}(S) \subseteq \operatorname{Fix}(T)$ and the operator $S$ has property $(\mathscr{A})$ with respect to the operator $T$.

We say $S: X \rightarrow C$ is a seed operator for computation of solutions of $\mathbf{F P P}(1)$.

Remark 3.1 If $S$ is an algorithmic operator for computation of solutions of $\mathbf{F P P}(1)$, the assumption (A0) holds automatically.

\subsection{The relaxed inertial Mann iteration method: RI-MIM}

In this section, we introduce and analyze the relaxed inertial Mann iteration method for computation of solutions of $\mathbf{F P P}(1)$ and study its weak convergence and convergence rate in a Hilbert space.

We introduce the relaxed inertial Mann iteration method (RI-MIM) for computation of solutions of $\mathbf{F P P}(1)$.

Algorithm 3.1 Let $C$ be a nonempty subset of $X$ and let $S: X \rightarrow C$ be a given operator such that $\operatorname{Fix}(S) \neq \emptyset$. Suppose that $T: X \rightarrow X$ is an algorithmic operator for problem $\boldsymbol{F P P}(1)$.

(i) Initialization: Select $x_{0}, x_{1} \in C$ arbitrarily.

(ii) Iterative step: Select sequences $\left\{\alpha_{n}\right\}$ in $\left(0, \alpha_{\max }\right)$ for some $\alpha_{\max } \in(1, \infty)$ and $\left\{\theta_{n}\right\}$ in $[0,1)$ and compute the $(n+1)^{\text {th }}$ iteration as follows:

$$
\left\{\begin{array}{l}
w_{n}=x_{n}+\theta_{n}\left(x_{n}-x_{n-1}\right) \\
x_{n+1}=\left(1-\alpha_{n}\right) w_{n}+\alpha_{n} T w_{n} \text { for all } n \in \mathbb{N}
\end{array}\right.
$$

For $\theta_{n}=0,(13)$ reduces to

$$
x_{n+1}=\left(1-\alpha_{n}\right) x_{n}+\alpha_{n} T x_{n} \text { for all } n \in \mathbb{N} .
$$

We say (14) is the relaxed Mann iteration method (RMIM).

Remark 3.2 (i) The orbit $\left\{x_{n}\right\}$ of Algorithm 3.1 is well defined without the convexity of $C$.

(ii) The initial points $x_{0}$ and $x_{1}$ can be also chosen from $X$.

(iii) In the papers $[17,41]$, authors studied convergence analysis of Mann-like iteration method for fixed points of nonexpansive operators in Hilbert and Banach spaces, respectively. Our approach is different from them.

Now our goal is to analyze the relaxed inertial Mann iteration method (13) when involved algorithmic operator is strongly quasi-nonexpansive. More precisely, for convergence analysis of Algorithm 3.1, we assume that the algorithmic operator $T: X \rightarrow X$ is $\kappa$-strongly quasi-nonexpansive and sequences $\left\{\alpha_{n}\right\}$ and $\left\{\theta_{n}\right\}$ satisfy the following conditions:

(C1) $0<a \leq \alpha_{n} \leq b<1+\kappa$ for all $n \in \mathbb{N}$ and for some $\kappa \in(0, \infty)$,

(C2) $\left\{\theta_{n}\right\}$ is non-decreasing sequence in $[0,1)$,

(C3) there exists $\theta \in(0,1)$ such that $\theta_{n} \leq \theta<\delta(\varepsilon)$ for all $n \in \mathbb{N}$, where $\delta$ is defined in (12) and $\varepsilon=\frac{1+\kappa-b}{b}$. 
In view of the condition $(\mathbf{C} 1)$, one can take $\alpha_{n}=1$ for all $n \in \mathbb{N}$. In this case, the relaxed inertial Mann iteration method (13) reduces to inertial Picard iteration method:

$$
x_{n+1}=T\left(x_{n}+\theta_{n}\left(x_{n}-x_{n-1}\right)\right) \text { for all } n \in \mathbb{N} .
$$

Remark 3.3 For $\alpha_{n}=b=1$ for all $n \in \mathbb{N}$, the condition (C3) reduces to the condition (C4):

(C4) there exists $\theta \in(0,1)$ such that $\theta_{n} \leq \theta<\delta(\kappa)$ for all $n \in \mathbb{N}$.

First, we study some basic properties of orbit of the relaxed inertial Mann iteration method (13) for solving $\mathbf{F P P}(1)$.

Proposition 3.1 Let $C$ be a nonempty subset of $X$ and $S: X \rightarrow C$ an operator such that $\operatorname{Fix}(S) \neq \emptyset$. Suppose that $T: X \rightarrow X$ is an algorithmic operator for $\boldsymbol{F P P}(1)$ such that $T$ is $\kappa$-strongly quasi-nonexpansive. For $x_{0}=x_{1} \in C$, let $\left\{x_{n}\right\}$ be a sequence in $X$ generated by the relaxed inertial Mann iteration method (13), where sequences $\left\{\alpha_{n}\right\}$ and $\left\{\theta_{n}\right\}$ satisfy the conditions (C1), (C2) and (C3). Then, for $v \in \operatorname{Fix}(S)$, the following hold:

(a) $\sum_{n=1}^{\infty}\left\|x_{n+1}-x_{n}\right\|^{2}<\infty$.

(b) $\lim _{n \rightarrow \infty}\left\|x_{n}-v\right\|$ and $\lim _{n \rightarrow \infty}\left\|w_{n}-v\right\|$ exist and $\lim _{n \rightarrow \infty}\left\|x_{n}-w_{n}\right\|=\lim _{n \rightarrow \infty}\left\|w_{n}-T w_{n}\right\|=0$.

(c) $\sum_{n=1}^{\infty}\left\|w_{n}-T w_{n}\right\|^{2}<\infty$ and

$$
R_{T,\left\{w_{n}\right\}}(n)=o\left(\frac{1}{\sqrt{n}}\right)
$$

We are now in the position to establish the convergence theorem for the relaxed inertial Mann iteration method (13).

Theorem 3.2 Let $C$ be a nonempty weakly closed subset of $X$ and $S: X \rightarrow C$ an operator such that $I-S$ is demiclosed at zero and $\operatorname{Fix}(S) \neq \emptyset$. Suppose that $T: X \rightarrow X$ is an algorithmic operator for $\boldsymbol{F P P}(1)$ such that $T$ is uniformly continuous and $\kappa$-strongly quasi-nonexpansive. For $x_{0}=x_{1} \in C$, let $\left\{x_{n}\right\}$ be a sequence in $X$ generated by the relaxed inertial Mann iteration method (13), where sequences $\left\{\alpha_{n}\right\}$ and $\left\{\theta_{n}\right\}$ satisfy the conditions (C1), (C2) and (C3). Then $\left\{x_{n}\right\}$ converges weakly to an element of $\operatorname{Fix}(S)$.

Proof Proposition 3.1 shows that $\lim _{n \rightarrow \infty}\left\|x_{n}-w_{n}\right\|=0$ and $\lim _{n \rightarrow \infty}\left\|w_{n}-T w_{n}\right\|=0$. By the uniform continuity of $T$, we have $\lim _{n \rightarrow \infty}\left\|x_{n}-T x_{n}\right\|=0$. Observe that

(i) $\lim _{n \rightarrow \infty}\left\|x_{n}-z\right\|$ exists for all $z \in \operatorname{Fix}(S)$,

(ii) $\lim _{n \rightarrow \infty}\left\|x_{n}-S x_{n}\right\|=0$ by the property $(\mathscr{A})$,

(iii) $I-S$ is demiclosed at zero.

Proposition 2.1 shows that $\left\{x_{n}\right\}$ converges weakly to an element of $\operatorname{Fix}(S)$.

We derive the following result in which $T$ is not necessarily uniformly continuous.

Corollary 3.1 Let $C$ be a nonempty weakly closed subset of $X$ and $S: X \rightarrow C$ an operator such that $I-S$ is demiclosed at zero and $\operatorname{Fix}(S) \neq \emptyset$. Suppose that $T: X \rightarrow X$ is an algorithmic operator for $\boldsymbol{F P P}(1)$ such that $T$ is $\kappa$-strongly quasi-nonexpansive. For $x_{1} \in C$, let $\left\{x_{n}\right\}$ be a sequence in $X$ generated by the relaxed Mann iteration method (14), where sequence $\left\{\alpha_{n}\right\}$ satisfies the condition $(\boldsymbol{C} 1)$. Then $\left\{x_{n}\right\}$ converges weakly to an element of $\operatorname{Fix}(S)$.

Proof For $v \in \operatorname{Fix}(S)$, from (54), we have

$$
\left\|x_{n+1}-v\right\|^{2} \leq\left\|x_{n}-v\right\|^{2}-\alpha_{n}\left(1+\kappa-\alpha_{n}\right)\left\|x_{n}-T x_{n}\right\|^{2} .
$$

Observe that $\lim _{n \rightarrow \infty}\left\|x_{n}-z\right\|$ exists for all $z \in \operatorname{Fix}(S)$ and $\lim _{n \rightarrow \infty}\left\|x_{n}-S x_{n}\right\|=0$ by the property $(\mathscr{A})$. Since $I-S$ is demiclosed at zero, therefore, we conclude from Proposition 2.1 that $\left\{x_{n}\right\}$ converges weakly to an element of $\operatorname{Fix}(S)$. 
3.2 The relaxed inertial normal S-iteration method: RInSIM

In this section, we introduce another relaxed inertial iterative method for computation of solutions of problem $\mathbf{F P P}(1)$ and study its weak convergence and convergence rate in a Hilbert space. This relaxed inertial iterative method is based on normal S-iteration method (NSIM) [25].

The normal S-iteration method (NSIM) was introduced by author [25] as follows:

$$
x_{n+1}=T\left[\left(1-\alpha_{n}\right) x_{n}+\alpha_{n} T x_{n}\right] \text { for all } n \in \mathbb{N},
$$

where $\left\{\alpha_{n}\right\}$ is real sequence in $(0,1)$ and $T: C \rightarrow C$ is a nonexpansive on a nonempty closed convex set $C$ of suitable Banach space. In recent years, S-iterative methodology is applied for solving various nonlinear problems, inclusion problems, optimization problems and image recovery problems (see [1, 2, 25, 26, 28,31,32]).

We now introduce the relaxed inertial normal S-iteration method (RI-NSIM) for computation of solutions of problem $\mathbf{F P P}(1)$.

Algorithm 3.3 Let $C$ be a nonempty subset of $X$ and let $S: X \rightarrow C$ be a given operator such that $\operatorname{Fix}(S) \neq \emptyset$. Suppose that $T: X \rightarrow X$ is an algorithmic operator for problem $\boldsymbol{F P P}(1)$.

(i) Initialization: Select $x_{0}, x_{1} \in C$ arbitrarily.

(ii) Iterative step: Select sequences $\left\{\alpha_{n}\right\}$ in $\left(0, \alpha_{\max }\right)$ for some $\alpha_{\max } \in(1, \infty)$ and $\left\{\theta_{n}\right\}$ in $[0,1)$ and compute the $(n+1)^{\text {th }}$ iteration as follows:

$$
\left\{\begin{array}{l}
w_{n}=x_{n}+\theta_{n}\left(x_{n}-x_{n-1}\right) \\
x_{n+1}=T\left[\left(1-\alpha_{n}\right) w_{n}+\alpha_{n} T w_{n}\right] \text { for all } n \in \mathbb{N}
\end{array}\right.
$$

For $\theta_{n}=0$ for all $n \in \mathbb{N}$, the relaxed inertial normal S-iteration method (18) reduces to

$$
x_{n+1}=T\left[\left(1-\alpha_{n}\right) x_{n}+\alpha_{n} T x_{n}\right] \text { for all } n \in \mathbb{N},
$$

where $\left\{\alpha_{n}\right\}$ is a sequence in $\left(0, \alpha_{\max }\right)$ for some $\alpha_{\max } \in(1, \infty)$. We say (19) is the relaxed normal S-iteration method.

Remark 3.4 The relaxed normal S-iteration method (19) is new from normal S-iteration method (17) in the following contexts:

(i) the iteration parameter $\left\{\alpha_{n}\right\}$ is lying on interval $\left(0, \alpha_{\max }\right)$,

(ii) the given operator $\mathrm{S}$ is not involved in Algorithm (19).

We now study some basic properties of orbit of the relaxed inertial normal iteration method (18) for solving $\mathbf{F P P}(1)$.

Proposition 3.2 Let $C$ be a nonempty subset of $X$ and $S: X \rightarrow C$ an operator such that $\operatorname{Fix}(S) \neq \emptyset$. Suppose that $T: X \rightarrow X$ is an algorithmic operator for $\boldsymbol{F P P}(1)$ such that $T$ is $\beta$ Lipschitz continuous and $\kappa$-strongly quasi-nonexpansive. For $x_{0}=x_{1} \in C$, let $\left\{x_{n}\right\}$ be a sequence in $X$ generated by the relaxed inertial normal iteration method (18), where sequences $\left\{\alpha_{n}\right\}$ and $\left\{\theta_{n}\right\}$ satisfy the conditions $(\boldsymbol{C} 1),(\boldsymbol{C} 2)$ and $(\boldsymbol{C} 5)$ :

(C5) there exists $\theta \in(0,1)$ such that $\theta_{n} \leq \theta<\delta(\mathcal{E})$ for all $n \in \mathbb{N}$, where $\delta$ is defined in (12) and

$$
\mathcal{E}=\frac{a(1+\kappa-b)}{2\left(1+b^{2} \beta^{2}\right)} .
$$

Then, for $v \in \operatorname{Fix}(S)$, the following hold:

(a) $\sum_{n=1}^{\infty}\left\|x_{n+1}-x_{n}\right\|^{2}<\infty$.

(b) $\lim _{n \rightarrow \infty}\left\|x_{n}-v\right\|$ and $\lim _{n \rightarrow \infty}\left\|w_{n}-v\right\|$ exist and $\lim _{n \rightarrow \infty}\left\|x_{n}-w_{n}\right\|=\lim _{n \rightarrow \infty}\left\|w_{n}-T w_{n}\right\|=0$.

(c) $\sum_{n=1}^{\infty}\left\|x_{n}-T x_{n}\right\|^{2}<\infty$ and

$$
R_{T,\left\{x_{n}\right\}}(n)=o\left(\frac{1}{\sqrt{n}}\right)
$$


Now, we are in a position to show our weak convergence theorem for the relaxed inertial normal S-iteration method (18).

Theorem 3.4 Let $C$ be a nonempty weakly closed subset of $X$ and $S: X \rightarrow C$ an operator such that $I-S$ is demiclosed at zero and $\operatorname{Fix}(S) \neq \emptyset$. Suppose that $T: X \rightarrow X$ is an algorithmic operator for problem $\boldsymbol{F P P}(1)$ such that $T$ is $\beta$-Lipschitz continuous and $\kappa$-strongly quasi-nonexpansive. For $x_{0}=x_{1} \in C$, let $\left\{x_{n}\right\}$ be a sequence in $X$ generated by the relaxed inertial normal $S$-iteration method (18), where $\left\{\alpha_{n}\right\}$ is a sequence in $(0, \infty)$ and $\left\{\theta_{n}\right\}$ is a sequence in $[0,1)$ satisfying the conditions $(\boldsymbol{C} 1),(\boldsymbol{C} 2)$ and $(\boldsymbol{C} 5)$. Then $\left\{x_{n}\right\}$ converges weakly to an element of $\operatorname{Fix}(S)$.

Proof Proposition 3.2(c) shows that $\lim _{n \rightarrow \infty}\left\|x_{n}-T x_{n}\right\|=0$. Observe that

(i) $\lim _{n \rightarrow \infty}\left\|x_{n}-z\right\|$ exists for all $z \in \operatorname{Fix}(S)$,

(ii) $\lim _{n \rightarrow \infty}\left\|x_{n}-S x_{n}\right\|=0$ by the property $(\mathscr{A})$,

(iii) $I-S$ is demiclosed at zero.

Proposition 2.1 shows that $\left\{x_{n}\right\}$ converges weakly to an element of $\operatorname{Fix}(S)$.

Corollary 3.2 Let $C$ be a nonempty weakly closed subset of $X$ and $S: X \rightarrow C$ an operator such that $I-S$ is demiclosed at zero and $\operatorname{Fix}(S) \neq \emptyset$. Suppose that $T: X \rightarrow X$ is an algorithmic operator for problem $\boldsymbol{F P P}(1)$ such that $T$ is $\beta$-Lipschitz continuous and $\kappa$-strongly quasi-nonexpansive. For $x_{1} \in C$, let $\left\{x_{n}\right\}$ be a sequence in $X$ generated by the relaxed normal $S$-iteration method (19), where $\left\{\alpha_{n}\right\}$ is a sequence in $(0, \infty)$ Then we have the following:

(a) If $\left\{\alpha_{n}\right\}$ satisfies the condition (C1), then $\left\{x_{n}\right\}$ converges weakly to an element of $\operatorname{Fix}(S)$.

(b) If $T$ is $\beta$-Lipschitz continuous and $0<\alpha_{n} \leq 1+\kappa$ for all $n \in \mathbb{N}$, then $\left\{x_{n}\right\}$ converges weakly to an element of $\operatorname{Fix}(S)$.

Proof For $v \in \operatorname{Fix}(S)$, from (61) (see, Appendix-III), we have

$$
\left\|x_{n+1}-v\right\|^{2} \leq\left\|x_{n}-v\right\|^{2}-\alpha_{n}\left(1+\kappa-\alpha_{n}\right)\left\|x_{n}-T x_{n}\right\|^{2}-\kappa\left\|y_{n}-T y_{n}\right\|^{2} .
$$

(a) Suppose that $\left\{\alpha_{n}\right\}$ satisfies the condition (C1). From (22), we get $\lim _{n \rightarrow \infty}\left\|x_{n}-z\right\|$ exists for all $z \in \operatorname{Fix}(S)$ and $\lim _{n \rightarrow \infty}\left\|x_{n}-S x_{n}\right\|=0$ by the property $(\mathscr{A})$. Since $I-S$ is demiclosed at zero, then Proposition 2.1 shows that $\left\{x_{n}\right\}$ converges weakly to an element of $\operatorname{Fix}(S)$.

(b) Suppose that $T$ is $\beta$-Lipschitz continuous and $0<\alpha_{n} \leq 1+\kappa$ for all $n \in \mathbb{N}$. For $v \in \operatorname{Fix}(S)$, from (22), we have

$$
\left\|x_{n+1}-v\right\|^{2} \leq\left\|x_{n}-v\right\|^{2}-\kappa\left\|y_{n}-T y_{n}\right\|^{2} .
$$

Thus, $\lim _{n \rightarrow \infty}\left\|x_{n}-z\right\|$ exists for all $z \in \operatorname{Fix}(S)$ and $\lim _{n \rightarrow \infty}\left\|y_{n}-T y_{n}\right\|=0$. From (64) (see Appendix-III), we obtain $\lim _{n \rightarrow \infty}\left\|x_{n}-T x_{n}\right\|=0$ and hence $\lim _{n \rightarrow \infty}\left\|x_{n}-S x_{n}\right\|=0$ by the property $(\mathscr{A})$. As Part (a), we see that $\left\{x_{n}\right\}$ converges weakly to an element of $\operatorname{Fix}(S)$.

We observe the following facts:

(i) Theorem 3.4 together with Proposition 3.2 establishes that the sequence $\left\{x_{n}\right\}$ generated by the relaxed inertial normal S-iteration method (18) converges weakly to an element of $\operatorname{Fix}(S)$ with the convergence rate given in $(21)$.

(ii) In view of Remark 3.4, Theorem 3.4 is new in the literature.

\subsection{Some deductions for nonexpansive type operators}

In view of Remark 3.1, we have

Theorem 3.5 Let $C$ be a nonempty weakly closed subset of $X$ and $T: X \rightarrow C$ a $\kappa$-strongly quasi-nonexpansive operator such that $I-T$ is demiclosed at zero. Let $x_{0}=x_{1} \in C$, let $\left\{x_{n}\right\}$ be a sequence in $X$ generated by the relaxed inertial Mann iteration method (13), where $\left\{\theta_{n}\right\}$ is a sequence in $[0,1)$ satisfying the conditions (C1), (C2) and (C3). Then $\left\{x_{n}\right\}$ converges weakly to an element of $\operatorname{Fix}(T)$. 
Proof Note that the assumption (A0) holds with $S=T$. Therefore, Theorem 3.5 follows from Theorem 3.2.

Similarly, from Theorem 3.4, we have

Theorem 3.6 Let $C$ be a nonempty weakly closed subset of $X$ and $T: X \rightarrow C$ a $\kappa$-strongly quasinonexpansive operator such that $T$ is $\beta$-Lipschitz continuous and $I-T$ is demiclosed at zero. For $x_{0}=x_{1} \in C$, let $\left\{x_{n}\right\}$ be a sequence in $X$ generated by the relaxed inertial normal S-iteration method (18), where $\left\{\alpha_{n}\right\}$ is a sequence in $(0, \infty)$ and $\left\{\theta_{n}\right\}$ is a sequence in $[0,1)$ satisfying the conditions $(\boldsymbol{C} 1),(\boldsymbol{C} 2)$ and $(\boldsymbol{C} 5)$. Then $\left\{x_{n}\right\}$ converges weakly to an element of $\operatorname{Fix}(T)$.

Remark 3.5 In author [26, Theorem 3.1] studied weak convergence the inertial Mann iteration method (13) for computation of fixed points of quasi-nonexpansive operators $T: C \rightarrow C$, where domain $C$ is necessarily affine. Our approach in Theorem 3.5 is different from [26, Theorem 3.1].

If the algorithmic operator $T$ is $\kappa$-strongly quasi-nonexpansive for solving $\mathbf{F P P}(1)$, then, in view of (C4) (see Remark 3.3) and Theorem 3.2, we have

Corollary 3.3 Let $C$ be a nonempty weakly closed subset of $X$ and $S: X \rightarrow C$ an operator such that $I-S$ is demiclosed at zero and $\operatorname{Fix}(S) \neq \emptyset$. Suppose that $T: X \rightarrow X$ is an algorithmic operator for $\boldsymbol{F P P}(1)$ such that $T$ is $\kappa$-strongly quasi-nonexpansive. For $x_{0}=x_{1} \in C$, let $\left\{x_{n}\right\}$ be a sequence in $X$ generated by the inertial Picard iteration method (15), where $\left\{\theta_{n}\right\}$ is non-decreasing sequence in $[0,1)$ such that there exists $\theta \in(0,1)$ such that $\theta_{n} \leq \theta<\delta(\kappa)$ for all $n \in \mathbb{N}$. Then $\left\{x_{n}\right\}$ converges weakly to an element of $\operatorname{Fix}(S)$.

In particular, for the class of firmly nonexpansive operators, we have

Corollary 3.4 Let $T: X \rightarrow X$ be a firmly nonexpansive operator such that $\operatorname{Fix}(T) \neq \emptyset$. Assume that $\left\{\theta_{n}\right\}$ is non-decreasing sequence in $[0,1)$ and there exists $\theta \in(0,1)$ such that $\theta_{n} \leq \theta<\delta(1)$ for all $n \in \mathbb{N}$. Then, for $x_{0}=x_{1} \in C$, the sequence $\left\{x_{n}\right\}$ in $X$ generated by the inertial Picard iteration method (15) converges weakly to an element of $\operatorname{Fix}(T)$.

Corollary 3.5 Let $T: X \rightarrow X$ be a firmly nonexpansive operator such that $\operatorname{Fix}(T) \neq \emptyset$. For $x_{0}=x_{1} \in C$, let $\left\{x_{n}\right\}$ be a sequence in $X$ generated by the relaxed inertial normal S-iteration method (18), where $\left\{\alpha_{n}\right\}$ is a sequence in $(a, b)$ such that $0<a \leq \alpha_{n} \leq b<2$ for all $n \in \mathbb{N}$ and $\left\{\theta_{n}\right\}$ is a sequence in $[0,1)$ satisfying the condition (C2). Assume that there exists $\theta \in(0,1)$ such that $\theta_{n} \leq \theta<\delta(\mathcal{E})$ for all $n \in \mathbb{N}$, where $\delta$ is defined in (12) and $\mathcal{E}=\frac{a(2-b)}{2\left(1+b^{2}\right)}$. Then $\left\{x_{n}\right\}$ converges weakly to an element of $\operatorname{Fix}(T)$.

From Corollary 3.4, we derive the following

Corollary 3.6 $[4$, Proposition 2.1] Let $A: X \rightrightarrows X$ be a maximally monotone operator such that $A^{-1}(0) \neq \emptyset$. Assume that $\left\{\theta_{n}\right\}$ is non-decreasing sequence in $[0,1)$ and there exists $\theta \in(0,1)$ such that $\theta_{n} \leq \theta<1 / 3$ for all $n \in \mathbb{N}$. For $\lambda \in(0, \infty)$ and $x_{0}=x_{1} \in X$, let $\left\{x_{n}\right\}$ be a sequence in $X$ generated by the inertial proximal method:

$$
x_{n+1}=J_{\lambda}^{A}\left(x_{n}+\theta_{n}\left(x_{n}-x_{n-1}\right)\right) \text { for all } n \in \mathbb{N},
$$

where $\left\{\theta_{n}\right\}$ is a sequence in $[0,1)$ satisfying the conditions $(\boldsymbol{C} 2)$ and $(\boldsymbol{C} 3)$. Then $\left\{x_{n}\right\}$ converges weakly to an element of $A^{-1}(0)$.

From Corollary 3.5, we derive the following new result:

Theorem 3.7 Let $A: X \rightrightarrows X$ be a maximally monotone operator such that $A^{-1}(0) \neq \emptyset$. For $\lambda \in(0, \infty)$ and $x_{0}=x_{1} \in C$, let $\left\{x_{n}\right\}$ be a sequence in $X$ generated by the relaxed inertial normal S-iteration method:

$$
\left\{\begin{array}{l}
w_{n}=x_{n}+\theta_{n}\left(x_{n}-x_{n-1}\right) \\
x_{n+1}=J_{\lambda}^{A}\left[\left(1-\alpha_{n}\right) w_{n}+\alpha_{n} J_{\lambda}^{A} w_{n}\right] \text { for all } n \in \mathbb{N},
\end{array}\right.
$$

where $\left\{\alpha_{n}\right\}$ and $\left\{\theta_{n}\right\}$ satisfy all the corresponding assumptions of Corollary 3.5. Then $\left\{x_{n}\right\}$ converges weakly to an element of $A^{-1}(0)$. 


\section{Applications}

4.1 Applications to pseudo-monotone variational inequality problems

In a Hilbert space, the relationship between the variational inequality problem $\operatorname{VI}(C, F)$ and a fixed point problem can be made through the characterization of the projection operator $P_{C}$ as follows:

Lemma 4.1 Let $C$ be a nonempty closed convex subset of $X$ and $F: X \rightarrow X$ a given operator. Then $\Omega[\operatorname{VI}(C, F)]=\operatorname{Fix}\left(P_{C}(I-\mu F)\right)$ for all $\mu \in(0, \infty)$.

Let us recall the following result.

Lemma 4.2 (see [40]) Let $C$ be a nonempty closed convex subset of $X$. Let $F: X \rightarrow X$ be a hemi-continuous pseudo-monotone operator. Then, the variational inequality problem $\operatorname{VI}(C, F)$ :

$$
\text { find } x^{*} \in C \text { such that }\left\langle F x^{*}, x-x^{*}\right\rangle \geq 0 \text { for all } x \in C
$$

is equivalent to the dual variational inequality problem $\operatorname{DVI}(C, F)$ :

$$
\text { find } x^{*} \in C \text { such that }\left\langle F x, x-x^{*}\right\rangle \geq 0 \text { for all } x \in C \text {. }
$$

Proposition 4.1 Let $C$ be a nonempty closed convex subset of $X$. Let $F: X \rightarrow X$ be a hemicontinuous monotone operator satisfying the condition $(\mathscr{B})$ :

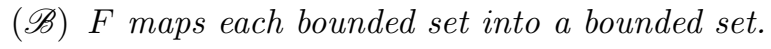

For $\lambda \in(0, \infty)$, define an operator $S_{\lambda}: X \rightarrow C$ by

$$
S_{\lambda}=P_{C}(I-\lambda F) \text {. }
$$

Then $I-S_{\lambda}$ is demiclosed at zero.

Proof Since $F$ is hemi-continuous monotone operator, from Lemma 4.2, we have

$$
x^{*} \in \Omega[\mathrm{VI}(C, F)] \Leftrightarrow\left\langle F x, x-x^{*}\right\rangle \geq 0 \text { for all } x \in C .
$$

Let $\left\{x_{n}\right\}$ be a sequence in $X$ such that $\left\{x_{n}\right\}$ converges weakly to $z$ and $\left\{\left(I-S_{\lambda}\right) x_{n}\right\}$ converges strongly to zero. We now show that $\left(I-S_{\lambda}\right) z=0$. Let $x \in C$. Set $y_{n}=S_{\lambda} x_{n}$. From Lemma 2.1(a), we have

$$
\begin{aligned}
\left\langle x_{n}-\lambda F x_{n}-y_{n}, x-y_{n}\right\rangle & =\left\langle x_{n}-\lambda F x_{n}-P_{C}(I-\lambda F) x_{n}, x-P_{C}(I-\lambda F) x_{n}\right\rangle \\
& \leq 0 \text { for all } n \in \mathbb{N} .
\end{aligned}
$$

Then, by the monotonicity of $F$, we have

$$
\begin{aligned}
\left\langle\lambda F x, x_{n}-x\right\rangle & =\lambda\left\langle F x_{n}, x_{n}-x\right\rangle-\lambda\left\langle F x_{n}-F x, x_{n}-x\right\rangle \\
& \leq \lambda\left\langle F x_{n}, x_{n}-x\right\rangle \\
& =\lambda\left\langle F x_{n}, x_{n}-y_{n}\right\rangle-\lambda\left\langle F x_{n}, x-y_{n}\right\rangle \\
& =\lambda\left\langle F x_{n}, x_{n}-y_{n}\right\rangle+\left\langle x_{n}-\lambda F x_{n}-y_{n}, x-y_{n}\right\rangle-\left\langle x_{n}-y_{n}, x-y_{n}\right\rangle \\
& \leq \lambda\left\langle F x_{n}, x_{n}-y_{n}\right\rangle-\left\langle x_{n}-y_{n}, x-y_{n}\right\rangle \\
& \leq\left(\lambda\left\|F x_{n}\right\|+\left\|y_{n}-x\right\|\right)\left\|x_{n}-y_{n}\right\|,
\end{aligned}
$$

which implies that

$$
\left\langle F x, x_{n}-x\right\rangle \leq\left(\left\|F x_{n}\right\|+\frac{1}{\lambda}\left\|y_{n}-x\right\|\right)\left\|x_{n}-y_{n}\right\| .
$$

Note $\left\{x_{n}\right\}$ is bounded. It follows, from the condition $(\mathscr{B})$ that $\left\{F x_{n}\right\}$ is bounded. Since $x_{n} \rightarrow z$ and $x_{n}-y_{n} \rightarrow 0$, we have $\langle F x, z-x\rangle \leq 0$. It follows, from (24), that $z \in \Omega[\operatorname{VI}(C, F)]$. Therefore, from Lemma 4.1, we have $z=P_{C}(I-\lambda F) z$. 
Proposition 4.2 Let $C$ be a nonempty closed convex subset of $X$. Let $F: X \rightarrow X$ be a hemicontinuous pseudo-monotone operator satisfying the condition $(\mathscr{B})$. For $\lambda \in(0, \infty)$, define an operator $S_{\lambda}: X \rightarrow C$ by (23). Suppose that $F$ is sequentially weak-to-weak continuous. Then $I-S_{\lambda}$ is demiclosed at zero.

Remark 4.1 If $F$ is $L$-Lipschitz continuous, then we have the following:

(i) $F$ satisfies the condition $(\mathscr{B})$,

(ii) the operator $S_{\lambda}$ defined by (23) is $(1+\lambda L)$-Lipschitz continuous. Indeed, for $u, v \in X$, we have

$$
\left\|S_{\lambda} u-S_{\lambda} v\right\| \leq\|u-v-\lambda(F u-F v)\| \leq(1+\lambda L)\|u-v\|,
$$

(iii) in Propositions 4.1 and 4.2 , both hemi-continuity of $F$ and condition $(\mathscr{B})$ are satisfied.

Let $C$ be a nonempty closed convex subset of $X$ and $F: X \rightarrow X$ a pseudo-monotone operator. For computational theory of solutions of $\mathrm{VI}(C, F)$, we consider three extragradient operators as below:

For $\lambda \in(0, \infty)$, following [6], we define operators $E_{\lambda}^{F} \equiv E_{\lambda}: X \rightarrow C, T_{\lambda}^{F} \equiv T_{\lambda}: X \rightarrow X$ and $U_{\lambda}^{F} \equiv U_{\lambda}: X \rightarrow X$ by

$$
\begin{gathered}
E_{\lambda}(x)=P_{C}\left(x-\lambda F S_{\lambda}(x)\right) \text { for all } x \in X, \\
T_{\lambda} x=P_{C}(x-\lambda F x)-\lambda\left(F S_{\lambda}(x)-F x\right) \text { for all } x \in X
\end{gathered}
$$

and

$$
U_{\lambda} x=P_{H_{x}}\left(x-\lambda F S_{\lambda}(x)\right) \text { for all } x \in X,
$$

respectively, where $S_{\lambda}: X \rightarrow C$ is an operator defined by (23) and $H_{x}=\{w \in X:\langle x-\lambda F x-$ $\left.\left.S_{\lambda}(x), w-S_{\lambda}(x)\right\rangle \leq 0\right\}$.

We now explore some basic properties of extragradient operators $E_{\lambda}, T_{\lambda}$ and $U_{\lambda}$. Specially, we show that all extragradient operators $E_{\lambda}, T_{\lambda}$ and $U_{\lambda}$ are strongly quasi-nonexpansive.

Proposition 4.3 Let $C$ be a nonempty closed convex subset of $X$ and $F: X \rightarrow X$ a pseudomonotone and L-Lipschitz continuous such that $\Omega[\mathrm{VI}(C, F)] \neq \emptyset$. For $\lambda \in(0, \infty)$, let $E_{\lambda}: X \rightarrow C$ be an extragradient operator defined by (25). Then, we have the following:

(a) $\left\|E_{\lambda}(u)-E_{\lambda}(v)\right\| \leq\left(1+\lambda L+\lambda^{2} L^{2}\right)\|u-v\|$ for all $u, v \in X$.

(b) For $\lambda \in(0,1 / L), x \in X$ and $v \in \Omega[\operatorname{VI}(C, F)]$, we have

(i) $\left\|E_{\lambda}(x)-v\right\|^{2} \leq\|x-v\|^{2}-(1-\lambda L)\left\|x-S_{\lambda}(x)\right\|^{2}-(1-\lambda L)\left\|S_{\lambda}(x)-E_{\lambda}(x)\right\|^{2}$.

(ii) $\left\|E_{\lambda}(x)-v\right\|^{2} \leq\|x-v\|^{2}-\frac{1-\lambda L}{2}\left\|x-E_{\lambda}(x)\right\|^{2}$.

(c) $\operatorname{Fix}\left(S_{\lambda}\right)=\operatorname{Fix}\left(E_{\lambda}\right)=\Omega[\operatorname{VI}(C, F)]$.

(d) For $\lambda \in(0,1 / L)$, the operator $S_{\lambda}$ has property $(\mathscr{A})$ with respect to the operator $E_{\lambda}$.

Proposition 4.4 Let $C$ be a nonempty closed convex subset of $X$ and $F: X \rightarrow X$ a pseudomonotone and L-Lipschitz continuous operator such that

$\Omega[\operatorname{VI}(C, F)] \neq \emptyset$. For $\lambda \in(0, \infty)$, define an operator $T_{\lambda}: X \rightarrow X$ by (26). Then we have the following:

(a) $\left\|T_{\lambda} u-T_{\lambda} v\right\| \leq \beta\|u-v\|$ for all $u, v \in X$, where $\beta=1+3 \lambda L+\lambda^{2} L^{2}$.

(b) For $\lambda \in(0,1 / L), x \in X$ and $z \in \Omega[\mathrm{VI}(C, F)]$, we have

(i) $\left\|x-T_{\lambda} x\right\| \leq(1+\lambda L)\left\|x-S_{\lambda} x\right\|$,

(ii) $(1-\lambda L)\left\|x-S_{\lambda} x\right\| \leq\left\|x-T_{\lambda} x\right\|$,

(iii) $\left\|T_{\lambda} x-z\right\|^{2} \leq\|x-z\|^{2}-\left(1-\lambda^{2} L^{2}\right)\left\|x-S_{\lambda} x\right\|^{2}$,

(iv) $\left\|T_{\lambda} x-z\right\|^{2} \leq\|x-z\|^{2}-\kappa\left\|x-T_{\lambda} x\right\|^{2}$, where

$$
\kappa=(1-\lambda L) /(1+\lambda L) .
$$

(c) $\operatorname{Fix}\left(S_{\lambda}\right)=\Omega[\mathrm{VI}(C, F)]=\operatorname{Fix}\left(T_{\lambda}\right)$.

(d) For $\lambda \in(0,1 / L)$, the operator $S_{\lambda}$ has property $(\mathscr{A})$ with respect to the operator $T_{\lambda}$. 
Proposition 4.5 Let $C$ be a nonempty closed convex subset of $X$ and $F: X \rightarrow X$ a pseudomonotone and L-Lipschitz continuous operator such that $\Omega[\operatorname{VI}(C, F)] \neq \emptyset$. For $\lambda \in(0, \infty)$, define operator $U_{\lambda}: X \rightarrow X$ by (27). Then, we have the following:

(a) $\left\|U_{\lambda}(u)-U_{\lambda}(v)\right\| \leq\left(1+\lambda L+\lambda^{2} L^{2}\right)\|u-v\|$ for all $u, v \in X$.

(b) For $\lambda \in(0,1 / L), x \in X$ and $v \in \Omega[\operatorname{VI}(C, F)]$, we have

(i) $\left\|U_{\lambda}(x)-v\right\|^{2} \leq\|x-v\|^{2}-(1-\lambda L)\left\|x-S_{\lambda}(x)\right\|^{2}-(1-\lambda L)\left\|S_{\lambda}(x)-U_{\lambda}(x)\right\|^{2}$.

(ii) $\left\|U_{\lambda}(x)-v\right\|^{2} \leq\|x-v\|^{2}-\frac{1-\lambda L}{2}\left\|x-U_{\lambda}(x)\right\|^{2}$.

(c) $\operatorname{Fix}\left(S_{\lambda}\right)=\operatorname{Fix}\left(U_{\lambda}\right)=\Omega[\operatorname{VI}(C, F)]$.

(d) For $\lambda \in(0,1 / L)$, the operator $S_{\lambda}$ has property $(\mathscr{A})$ with respect to the operator $U_{\lambda}$.

Proof Let $\lambda \in(0,1 / L), x \in X$ and $v \in \Omega[\operatorname{VI}(C, F)]$. Set $y=P_{C}(x-\lambda F x)$. Since $y \in C$, we have $\langle F v, y-v\rangle \geq 0$. By the pseudo-monotonicity of $F$, we have $\langle F y, y-v\rangle \geq 0$. Hence

$$
\left\langle F y, U_{\lambda}(x)-v\right\rangle=\left\langle F y, U_{\lambda}(x)-y\right\rangle+\langle F y, y-v\rangle \geq\left\langle F y, U_{\lambda}(x)-y\right\rangle .
$$

By the definition of $H_{x}$, we get

$$
\begin{aligned}
\left\langle x-y-\lambda F y, y-U_{\lambda}(x)\right\rangle & =\left\langle x-y-\lambda F x, y-U_{\lambda}(x)\right\rangle+\lambda\left\langle F x-F y, y-U_{\lambda}(x)\right\rangle \\
& \leq \lambda\left\langle F x-F y, y-U_{\lambda}(x)\right\rangle \\
& \leq \lambda\|F x-F y\|\left\|y-U_{\lambda}(x)\right\| \\
& \leq \frac{\lambda L}{2}\left(\|x-y\|^{2}+\left\|y-U_{\lambda}(x)\right\|^{2}\right) .
\end{aligned}
$$

From (27) and (29), we have

$$
\begin{aligned}
\left\|U_{\lambda}(x)-v\right\|^{2} \leq & \|x-\lambda F y-v\|^{2}-\left\|x-\lambda F y-P_{H_{x}}(x-\lambda F y)\right\|^{2} \\
= & \|x-\lambda F y-v\|^{2}-\left\|x-\lambda F y-U_{\lambda}(x)\right\|^{2} \\
= & \|x-v\|^{2}-2 \lambda\langle F y, x-v\rangle+\lambda^{2}\|F y\|^{2} \\
& -\left\|x-U_{\lambda}(x)\right\|^{2}+2 \lambda\left\langle F y, x-U_{\lambda}(x)\right\rangle-\lambda^{2}\|F y\|^{2} \\
= & \|x-v\|^{2}-\left\|x-U_{\lambda}(x)\right\|^{2}+2 \lambda\left\langle F y, v-U_{\lambda}(x)\right\rangle \\
\leq & \|x-v\|^{2}-\left\langle x-y+y-U_{\lambda}(x), x-y+y-U_{\lambda}(x)\right\rangle+2 \lambda\left\langle F y, y-U_{\lambda}(x)\right\rangle \\
= & \|x-v\|^{2}-\|x-y\|^{2}-\left\|y-U_{\lambda}(x)\right\|^{2}-2\left\langle x-y, y-U_{\lambda}(x)\right\rangle+2 \lambda\left\langle F y, y-U_{\lambda}(x)\right\rangle \\
= & \|x-v\|^{2}-\|x-y\|^{2}-\left\|y-U_{\lambda}(x)\right\|^{2}-2\left\langle x-y-\lambda F y, U_{\lambda}(x)-y\right\rangle .
\end{aligned}
$$

Hence, from (30), we get

$$
\left\|U_{\lambda}(x)-v\right\|^{2} \leq\|x-v\|^{2}-(1-\lambda L)\|x-y\|^{2}-(1-\lambda L)\left\|y-U_{\lambda}(x)\right\|^{2} .
$$

All other results of Proposition 4.5 are true as Proposition 4.3.

Remark 4.2 (i) In the light of Lemma 4.1, Propositions 4.3, 4.4 and 4.5, we observe that the operators $E_{\lambda}: X \rightarrow X, T_{\lambda}: X \rightarrow X$ and $U_{\lambda}: X \rightarrow X$ are algorithmic operators for computation of solutions of $\mathbf{F P P}(1)$ with $S=S_{\lambda}$.

(ii) All three algorithmic operators $E_{\lambda}, T_{\lambda}$ and $U_{\lambda}$ are in the class of strongly quasi-nonexpansive operators, which settles down the unification of extragradient methods $\operatorname{KEM}(4), \operatorname{TEM}(7)$ and $\operatorname{SEM}(8)$ for pseudo-monotone variational inequality problems. This provides an affirmative answer of (Q1).

In view of Remark 4.2(i), various inertial and non-inertial extragradient methods for solving the variational inequality problem $\mathrm{VI}(C, F)$ can be derived by applying fixed point algorithms (see $[2,23,25,26,28])$.

On the basis of the relaxed inertial Mann iteration method (13), inertial Picard iteration method (15), and the relaxed inertial normal S-iteration method (18) studied in Section 3, first 
we derive the following extragradient methods generated by algorithmic operator $E_{\lambda}$ for solving the problem $\mathrm{VI}(C, F)$ :

$$
\begin{aligned}
& \left\{\begin{array}{l}
w_{n}=x_{n}+\theta_{n}\left(x_{n}-x_{n-1}\right), \\
x_{n+1}=\left(1-\alpha_{n}\right) w_{n}+\alpha_{n} E_{\lambda} w_{n} \text { for all } n \in \mathbb{N},
\end{array}\right. \\
& x_{n+1}=E_{\lambda}\left(x_{n}+\theta_{n}\left(x_{n}-x_{n-1}\right)\right) \text { for all } n \in \mathbb{N},
\end{aligned}
$$

and

$$
\left\{\begin{array}{l}
w_{n}=x_{n}+\theta_{n}\left(x_{n}-x_{n-1}\right), \\
x_{n+1}=E_{\lambda}\left[\left(1-\alpha_{n}\right) w_{n}+\alpha_{n} E_{\lambda} w_{n}\right] \text { for all } n \in \mathbb{N},
\end{array}\right.
$$

respectively.

We will say:

(i) (31) is the relaxed inertial Mann iteration based Korpelevich's extragradient method (RIMKEM)

(ii) (32) is inertial Korpelevich's extragradient method (I-KEM),

(iii) (33) is the relaxed inertial normal S-iteration based Korpelevich's extragradient method (RInS-KEM).

Further, from iterative methods (13), (15), and (18), we derive the following extragradient methods generated by algorithmic operator $T_{\lambda}$ :

$$
\begin{aligned}
& \left\{\begin{array}{l}
w_{n}=x_{n}+\theta_{n}\left(x_{n}-x_{n-1}\right), \\
x_{n+1}=\left(1-\alpha_{n}\right) w_{n}+\alpha_{n} T_{\lambda} w_{n} \text { for all } n \in \mathbb{N},
\end{array}\right. \\
& x_{n+1}=T_{\lambda}\left(x_{n}+\theta_{n}\left(x_{n}-x_{n-1}\right)\right) \text { for all } n \in \mathbb{N},
\end{aligned}
$$

and

$$
\left\{\begin{array}{l}
w_{n}=x_{n}+\theta_{n}\left(x_{n}-x_{n-1}\right), \\
x_{n+1}=T_{\lambda}\left[\left(1-\alpha_{n}\right) w_{n}+\alpha_{n} T_{\lambda} w_{n}\right] \text { for all } n \in \mathbb{N}
\end{array}\right.
$$

respectively.

We will say:

(i) (34) is the relaxed inertial Mann iteration based Teng's extragradient method (RIM-TEM),

(ii) (35) is inertial Teng's extragradient method (I-TEM), TEM).

(iii) (36) is the relaxed inertial normal S-iteration based Tseng's extragradient method (RInS-

Similarly, from iterative methods (13), (15), and (18), we derive the following extragradient methods generated by algorithmic operator $U_{\lambda}$ :

$$
\begin{aligned}
& \left\{\begin{array}{l}
w_{n}=x_{n}+\theta_{n}\left(x_{n}-x_{n-1}\right), \\
x_{n+1}=\left(1-\alpha_{n}\right) w_{n}+\alpha_{n} U_{\lambda} w_{n} \text { for all } n \in \mathbb{N},
\end{array}\right. \\
& x_{n+1}=U_{\lambda}\left(x_{n}+\theta_{n}\left(x_{n}-x_{n-1}\right)\right) \text { for all } n \in \mathbb{N},
\end{aligned}
$$

and

$$
\left\{\begin{array}{l}
w_{n}=x_{n}+\theta_{n}\left(x_{n}-x_{n-1}\right), \\
x_{n+1}=U_{\lambda}\left[\left(1-\alpha_{n}\right) w_{n}+\alpha_{n} U_{\lambda} w_{n}\right] \text { for all } n \in \mathbb{N}
\end{array}\right.
$$

respectively.

We will say:

(i) (37) is the relaxed inertial Mann iteration based subgradient extragradient method (RIMSEM),

(ii) (38) is inertial subgradient extragradient method (I-SEM),

(iii) (39) is the relaxed inertial normal S-iteration based subgradient extragradient method (RInS-SEM).

Remark 4.3 For $\theta=0$, one can obtain corresponding non-inertial methods of (31), (33), (34), (36), (37) and (39). 
Now we are ready to study convergence analysis of extragradient methods (31), (33), (34), (36), (37) and (39) for solving $\mathrm{VI}(C, F)$.

Theorem 4.1 Let $C$ be a nonempty closed convex subset of $X$ and $F: X \rightarrow X$ a pseudo-monotone and L-Lipschitz continuous operator such that $\Omega[\mathrm{VI}(C, F)] \neq \emptyset$. For $\lambda \in(0,1 / L)$ and $x_{0}=x_{1} \in C$, let $\left\{x_{n}\right\}$ be a sequence in $X$ generated by RIM-KEM (31) or RIM-SEM (37), where sequences $\left\{\alpha_{n}\right\}$ and $\left\{\theta_{n}\right\}$ satisfy the conditions $(\boldsymbol{C} 1),(\boldsymbol{C} 2)$ and $(\boldsymbol{C} 3)$ with $\kappa=(1-\lambda L) / 2$. Then $\left\{x_{n}\right\}$ converges weakly to an element of $\Omega[\mathrm{VI}(C, F)]$.

Proof Let $S_{\lambda}: X \rightarrow C$ be an operator define by (23). From Proposition 4.3(c), we see that $\operatorname{Fix}\left(S_{\lambda}\right)=\Omega[\mathrm{VI}(C, F)]$. Proposition 4.2 infers that $I-S_{\lambda}$ is demiclosed at zero. For RIM-KEM (31), we observe that

(i) there exists the extragradient operator $E_{\lambda}$ defined by (25), which is $\kappa$-strongly quasi-nonexpansive with $\kappa=(1-\lambda L) / 2$ by Proposition 4.3 (b)(ii),

(ii) $\operatorname{Fix}\left(S_{\lambda}\right)=\operatorname{Fix}\left(E_{\lambda}\right)$ by Proposition 4.3 (c),

(iii) the operator $S_{\lambda}$ has property $(\mathscr{A})$ with respect to the operator $E_{\lambda}$ by Proposition 4.3 (d).

Thus, for $\mathbf{F P P}(1)$ with $S=S_{\lambda}$, all the assumptions of Theorem 3.2 holds. Therefore, Theorem 4.1 follows from Theorem 3.2.

Similarly, $\left\{x_{n}\right\}$, defined by RIM-SEM (37), converges weakly to an element of $\Omega[\operatorname{VI}(C, F)]$.

Theorem 4.2 Let $C$ be a nonempty closed convex subset of $X$ and $F: X \rightarrow X$ a pseudo-monotone and L-Lipschitz continuous operator such that $\Omega[\mathrm{VI}(C, F)] \neq \emptyset$. Let $\left\{\theta_{n}\right\}$ be a sequence in $[0,1)$ satisfying the condition (C2) and there exists $\theta \in(0,1)$ such that $\theta_{n} \leq \theta<1 / 3$ for all $n \in \mathbb{N}$. For $\lambda \in(0,1 / L)$ and $x_{0}=x_{1} \in C$, let $\left\{x_{n}\right\}$ be a sequence in $X$ generated by I-KEM (32) or I-SEM (38). Then $\left\{x_{n}\right\}$ converges weakly to an element of $\Omega[\mathrm{VI}(C, F)]$.

Theorem 4.3 Let $C$ be a nonempty closed convex subset of $X$ and $F: X \rightarrow X$ a pseudo-monotone and L-Lipschitz continuous operator such that

$\Omega[\operatorname{VI}(C, F)] \neq \emptyset$. For $\lambda \in(0,1 / L)$ and $x_{0}=x_{1} \in C$, let $\left\{x_{n}\right\}$ be a sequence in $X$ generated by RInS-KEM (33) or RInS-SEM (39), where sequences $\left\{\alpha_{n}\right\}$ and $\left\{\theta_{n}\right\}$ satisfy the conditions (C1), (C2) and (C5) with $\kappa=(1-\lambda L) / 2$. Then $\left\{x_{n}\right\}$ converges weakly to an element of $\Omega[\operatorname{VI}(C, F)]$.

Proof Note that $E_{\lambda}$ and $U_{\lambda}$ are $\left(1+\lambda L+\lambda^{2} L^{2}\right)$-Lipschitz continuous by Propositions 4.3 and 4.5, respectively. Therefore, Theorem 4.3 follows from Theorem 3.4.

Next, we drive convergence theorems for solving $\operatorname{VI}(C, F)$ when algorithmic operator is $T_{\lambda}$.

Theorem 4.4 Let $C$ be a nonempty closed convex subset of $X$ and $F: X \rightarrow X$ a pseudo-monotone and L-Lipschitz continuous operator such that $\Omega[\mathrm{VI}(C, F)] \neq \emptyset$. For $\lambda \in(0,1 / L)$ and $x_{0}=x_{1} \in C$, let $\left\{x_{n}\right\}$ be a sequence in $X$ generated by RIM-TEM (34), where sequences $\left\{\alpha_{n}\right\}$ and $\left\{\theta_{n}\right\}$ satisfy the conditions $(\boldsymbol{C} 1),(\boldsymbol{C} 2)$ and $(\boldsymbol{C} 3)$, where $\kappa$ is given by (28). Then $\left\{x_{n}\right\}$ converges weakly to an element of $\Omega[\mathrm{VI}(C, F)]$.

Proof Note that $I-S_{\lambda}$ is demiclosed at zero by Proposition 4.2 and $\operatorname{Fix}\left(S_{\lambda}\right)=\Omega[\operatorname{VI}(C, F)]$. Observe that

(i) there exists the extragradient operator $T_{\lambda}$ defined by (25), which is $\kappa$-strongly quasi-nonexpansive with $\kappa=(1-\lambda L) /(1+\lambda L)$ by Proposition 4.4 (b)(iv),

(ii) $\operatorname{Fix}\left(S_{\lambda}\right)=\operatorname{Fix}\left(T_{\lambda}\right)$ by Proposition $4.4(\mathrm{c})$,

(iii) the operator $S_{\lambda}$ has property $(\mathscr{A})$ with respect to the operator $T_{\lambda}$ by Proposition 4.4 (d).

Thus, for $\mathbf{F P P}(1)$ with $S=S_{\lambda}$, all the assumptions of Theorem 3.2 holds. Therefore, Theorem 4.1 follows from Theorem 3.2. 
Theorem 4.5 Let $C$ be a nonempty closed convex subset of $X$ and $F: X \rightarrow X$ a pseudo-monotone and L-Lipschitz continuous operator such that $\Omega[\operatorname{VI}(C, F)] \neq \emptyset$. Let $\left\{\theta_{n}\right\}$ be a sequence in $[0,1)$ satisfying the condition (C2) and there exists $\theta \in(0,1)$ such that $\theta_{n} \leq \theta<1 / 3$ for all $n \in \mathbb{N}$. For $\lambda \in(0,1 / L)$ and $x_{0}=x_{1} \in C$, let $\left\{x_{n}\right\}$ be a sequence in $X$ generated by I-TEM (35). Then $\left\{x_{n}\right\}$ converges weakly to an element of $\Omega[\mathrm{VI}(C, F)]$.

Remark 4.4 Thong and Hieu [34] studied weak convergence of I-TEM (35) for solving VI $(C, F)$ under the condition:

$$
\theta_{n} \leq \theta<\frac{\sqrt{1+8 \varepsilon}-(1+2 \varepsilon)}{2(1-\varepsilon)} \text { for all } n \in \mathbb{N},
$$

where $F$ is a monotone and $L$-Lipschitz continuous operator and $\varepsilon=\frac{1-\lambda L}{1+\lambda L}$. Clearly, the condition (40) depends on the constants $\lambda$ and $L$. Theorem 4.5 shows the weak convergence of I-TEM (35) under the simpler condition:

$$
\theta_{n} \leq \theta<\frac{1}{3} \text { for all } n \in \mathbb{N},
$$

which is independent from the constants $\lambda$ and $L$.

Theorem 4.6 Let $C$ be a nonempty closed convex subset of $X$ and $F: X \rightarrow X$ a pseudo-monotone and L-Lipschitz continuous operator such that $\Omega[\mathrm{VI}(C, F)] \neq \emptyset$. For $\lambda \in(0,1 / L)$ and $x_{0}=x_{1} \in C$, let $\left\{x_{n}\right\}$ be a sequence in $X$ generated by $\operatorname{RInS}$-TEM (36), where sequences $\left\{\alpha_{n}\right\}$ and $\left\{\theta_{n}\right\}$ satisfy the conditions $(\boldsymbol{C} 1),(\boldsymbol{C} 2)$ and $(\boldsymbol{C} 5)$, where $\kappa$ is given by (28). Then $\left\{x_{n}\right\}$ converges weakly to an element of $\Omega[\mathrm{VI}(C, F)]$.

Proof Noticing that $T_{\lambda}$ is $\left(1+3 \lambda L+\lambda^{2} L^{2}\right)$-Lipschitz continuous by Proposition 4.4(a). Therefore, Theorem 4.6 follows from Theorem 3.4.

Remark 4.5 (i) Theorem 4.4 is the inertial version of Bot et al. [9, Theorem 3.1] whereas Theorem 4.6 is the inertial version of Bot et al. [9, Theorem 3.1] based on normal S-iteration method (17).

(ii) Theorem 4.4 and Theorem 4.6 are improvements upon Bot et al. [9, Theorem 3.1] and Thong and Hieu [34, Theorem 3.3] in the context of the relaxed inertial variants of their extragradient methods.

In view of Corollaries 3.1 and 3.2 and Remark 4.3, we have

Theorem 4.7 Let $C$ be a nonempty closed convex subset of $X$ and $F: X \rightarrow X$ a pseudomonotone and L-Lipschitz continuous operator such that $\Omega[\operatorname{VI}(C, F)] \neq \emptyset$. Let $\lambda \in(0,1 / L)$ and $\left\{\alpha_{n}\right\}$ a sequence satisfies the conditions $(\boldsymbol{C} 1)$. Then we have the following:

(a) If $\kappa=(1-\lambda L) / 2$ and $\left\{x_{n}\right\}$ is a sequence in $X$ generated by the relaxed Mann iteration based Korpelevich's extragradient method (RM-KEM):

$$
x_{n+1}=\left(1-\alpha_{n}\right) x_{n}+\alpha_{n} E_{\lambda} x_{n} \text { for all } n \in \mathbb{N}
$$

or generated by the relaxed normal S-iteration based Korpelevich's extragradient method (RnS$K E M)$ :

$$
x_{n+1}=E_{\lambda}\left[\left(1-\alpha_{n}\right) x_{n}+\alpha_{n} E_{\lambda} x_{n}\right] \text { for all } n \in \mathbb{N},
$$

then $\left\{x_{n}\right\}$ converges weakly to an element of $\Omega[\mathrm{VI}(C, F)]$.

(b) If $\kappa=(1-\lambda L) /(1+\lambda L)$ and $\left\{x_{n}\right\}$ is a sequence in $X$ generated by the relaxed Mann iteration based Tseng's extragradient method (RM-TEM):

$$
x_{n+1}=\left(1-\alpha_{n}\right) x_{n}+\alpha_{n} T_{\lambda} x_{n} \text { for all } n \in \mathbb{N}
$$

or generated by the relaxed normal S-iteration based Tseng's extragradient method (RnS-TEM):

$$
x_{n+1}=T_{\lambda}\left[\left(1-\alpha_{n}\right) x_{n}+\alpha_{n} T_{\lambda} x_{n}\right] \text { for all } n \in \mathbb{N},
$$

then $\left\{x_{n}\right\}$ converges weakly to an element of $\Omega[\mathrm{VI}(C, F)]$. 
Remark 4.6 (i) In RM-TEM(41) and RnS-TEM(42), we have $\alpha_{n}<1+\frac{1-\lambda L}{1+\lambda L}=\frac{2}{1+L \lambda}$. Our algorithm RM-TEM(41) is identical to Algorithm 3.1 of Bot et al. [9].

(ii) In Section 3, the rate of convergence of the relaxed inertial Mann iterative method is estimated as $R_{T,\left\{w_{n}\right\}}(n)=o\left(\frac{1}{\sqrt{n}}\right)$ whereas the rate of convergence of the relaxed inertial normal S-iterative method is estimated as $R_{T,\left\{x_{n}\right\}}(n)=o\left(\frac{1}{\sqrt{n}}\right)$. Note all three algorithmic operators $E_{\lambda}$, $T_{\lambda}$ and $U_{\lambda}$ are strongly quasi-nonexpansive operators. This provides an affirmative answer of (Q2) in the light of the convergence rates of the relaxed inertial Mann iterative method (13) and the relaxed inertial normal S-iterative method (18).

\subsection{Applications to pseudo-monotone variational inequality problems}

Let $G$ be a nonempty open set in $\mathbb{R}^{m}$ and $f: G \rightarrow \mathbb{R}$ be a differentiable function. Then $f$ called pseudo-convex on $G$, if for all $x, y \in G$ it holds:

$$
\langle\nabla f(x), y-x\rangle \geq 0 \Rightarrow f(y) \geq f(x) .
$$

It is well-known that $f$ is pseudo-convex on $G$ if and only if $\nabla f$ is pseudo-monotone on $G$.

Consider the pseudo-convex optimization problem:

$$
\min _{x \in C} f(x),
$$

where $f: G \subseteq \mathbb{R}^{m} \rightarrow \mathbb{R}$ is a differentiable function with Lipschitz continuous gradient which is also pseudo-convex on an open convex set $G$ and $C$ is a nonempty closed convex set of $G$.

Thus, the relaxed extragradient methods studied in Subsection 4.1 can be used to solve pseudoconvex optimization problem (43).

We now give an example of pseudo-convex function for numerical solutions of pseudo-convex optimization problem (43).

Example 4.1 Let $X=\mathbb{R}^{m}$ and $d \in(0, \infty)$. Define $f: X \rightarrow \mathbb{R}$ by

$$
f(x)=\frac{\|x\|^{2}}{\|x\|^{2}+d}, \quad x \in X .
$$

Then we have the following:

(a) $F(x)=\nabla f(x)=\frac{2 d}{\left(\|x\|^{2}+d\right)^{2}} x$ for all $x \in X$.

(b) $F$ is pseudo-monotone.

(c) For all $x, u \in X$, we have $\nabla F(x)(u)=\frac{2 d}{\left(\|x\|^{2}+d\right)^{4}}\left(\left(\|x\|^{2}+d\right)^{2} u-4\left(\|x\|^{2}+d\right)(\langle x, u\rangle) x\right)$.

(d) $\|\nabla F(x)\| \leq \Psi(\|x\|)$ for all $x \in X$, where $\Psi:[0, \infty) \rightarrow[0, \infty)$ is a function defined by

$$
\Psi(t)=\frac{2 d}{\left(t^{2}+d\right)^{3}}\left(5 t^{2}+d\right) \text { for all } t \geq 0 .
$$

We observe that $\Psi(t) \leq \frac{125}{54 d}$ for all $t \geq 0$. Thus, $F$ is $\frac{125}{54 d}$-Lipschitz continuous on $X$. The simulation of function $\Psi$ is given in Figure 1 for $d=0.8,1,1.2$.

\section{Numerical experiments}

In this section, numerical convergence behaviour of sequences $\left\{x_{n}\right\}$ defined by our Algorithms RIMKEM(31), RInS-KEM(33), RIM-KEM(34), RInS-TEM(36), RIM-SEM(37) and RInS-SEM(39) for solutions of the variational inequality problem $\operatorname{VI}(C, F)$, where $F$ is monotone /pseudo-monotone and L-Lipschitz continuous on suitable space $X$.

Following Sahu and Singh [33, Example 2.1], we have the following: 
Example 5.1 Let $F: \mathbb{R}^{3} \rightarrow \mathbb{R}^{3}$ be an operator defined by

$$
F\left(x_{1}, x_{2}, x_{3}\right)=\left(F_{1}\left(x_{1}, x_{2}, x_{3}\right), F_{2}\left(x_{1}, x_{2}, x_{3}\right), F_{3}\left(x_{1}, x_{2}, x_{3}\right)\right)
$$

with

$$
\begin{aligned}
& F_{1}\left(x_{1}, x_{2}, x_{3}\right)=x_{1}-s x_{2}+t x_{3}+\sin \left(x_{1}\right), F_{2}\left(x_{1}, x_{2}, x_{3}\right)=x_{2}-r x_{3}+s x_{1}+\sin \left(x_{2}\right), \\
& F_{3}\left(x_{1}, x_{2}, x_{3}\right)=x_{3}-t x_{1}+r x_{2}+\sin \left(x_{3}\right)
\end{aligned}
$$

for all $\left(x_{1}, x_{2}, x_{3}\right) \in \mathbb{R}^{3}$, where $r, s, t$ are real numbers. Then $F$ is monotone and L-Lipschitz continuous, where $L=\sqrt{3 \max \left\{4+r^{2}+s^{2}, 4+s^{2}+t^{2}, 4+t^{2}+r^{2}\right\}}$.

Example 5.2 Let $U, V: X \rightarrow X$ be bounded linear operators such that $U$ is self-adjoint and satisfying:

$$
\langle V x, x\rangle \geq 0 \text { and }\langle U x, x\rangle \geq \eta\|x\|^{2} \text { for all } x \in X .
$$

where $\eta>0$. Let $F: X \rightarrow X$ be an operator defined by

$$
F(x)=\left(e^{-\langle x, U x\rangle}+\alpha\right)(V x+q) \text { for all } x \in X,
$$

where $\alpha \geq 0$ and $q \in X$. Then $F$ is pseudo-monotonous and

$$
\|\nabla F(x)\| \leq 2 e^{-\eta\|x\|^{2}}\|U\|\|x\|(\|V\|\|x\|+\|p\|)+\left(e^{-\eta\|x\|^{2}}+\alpha\right)\|V\| \text { for all } x \in X .
$$

Example 5.3 The operator $F$ is defined by

$$
F(x):=M x+q \text { for all } x \in \mathbb{R}^{m},
$$

where $M=B B^{\top}+S+D$, and $B, S, D \in \mathbb{R}^{m \times m}$ are randomly generated matrices such that $S$ is skew-symmetric, $D$ is a positive definite diagonal matrix (hence the variational inequality has a unique solution) and $q=0$.

For numerical experiments, we fixed the feasible set $C$, which is described by $\left\{x \in \mathbb{R}^{m}:-5 \leq\right.$ $\left.x_{i} \leq 5, i=1,2, \ldots, m\right\}$ and $\lambda=.85 L$ with $\kappa_{E}=(1-\lambda L) / 2, \kappa_{T}=(1-\lambda L) /(1+\lambda L), \beta_{E}=$ $\left(1+\lambda L+\lambda^{2} L^{2}\right)$ and $\beta_{T}=\left(1+3 \lambda L+\lambda^{2} L^{2}\right)$. Also we fixed initial points $x_{0}=x_{1} \in C$ and iteration parameters $\alpha_{n}, \theta_{n}, \varepsilon$ (or $\mathcal{E}$ ) and error term $E(n)$ as follows:

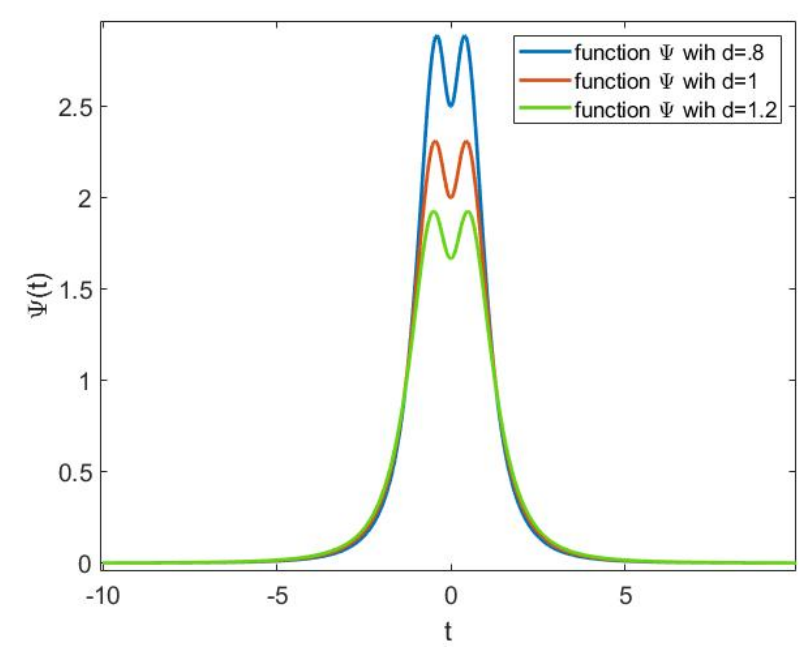

Fig. 1: The graph of $\Psi$ defined in Example 4.1 over $[-10,10]$ 
- $x_{0}=x_{1}=\frac{1}{\sqrt{m}}(1,1, \ldots, 1) \in \mathbb{R}^{m}$.

- $\alpha_{n}=b=.961\left(1+\kappa_{E}\right)$ and $\theta_{n}=.9 \delta(\varepsilon)$ for algorithms RIM-KEM(31) and RIM-SEM(37), where $\delta$ is defined in (12) and $\varepsilon=\left(1+\kappa_{E}-b\right) / b$.

- $\alpha_{n}=b=.961\left(1+\kappa_{E}\right)$ and $\theta_{n}=.9 \delta(\mathcal{E})$ in algorithms RInS-KEM(33) and RInS-SEM(39), where $\mathcal{E}=\frac{a\left(1+\kappa_{E}-b\right)}{2\left(1+b^{2} \beta_{E}^{2}\right)}$.

- $\alpha_{n}=b=.961\left(1+\kappa_{T}\right)$ and $\theta_{n}=.9 \delta(\varepsilon)$ for algorithm RIM-TEM(34), where $\varepsilon=\left(1+\kappa_{T}-b\right) / b$.

- $\alpha_{n}=b=.961\left(1+\kappa_{T}\right)$ and $\theta_{n}=.9 \delta(\mathcal{E})$ in algorithm IRnS-TEM(36), where $\mathcal{E}=\frac{a\left(1+\kappa_{T}-b\right)}{2\left(1+b^{2} \beta_{T}^{2}\right)}$.

- Define the error term

$$
E(n)=\left\|x_{n}-P_{C}(I-\lambda) x_{n}\right\|^{2} \leq \epsilon \text { for all } n \in \mathbb{N} .
$$

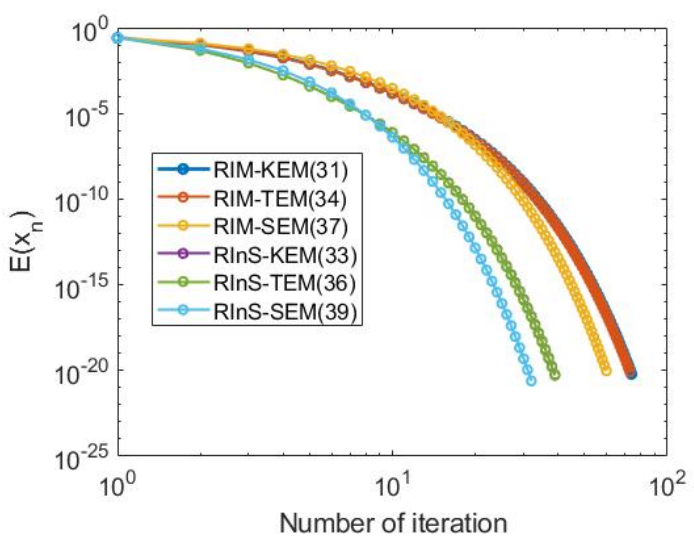

(a) when $\epsilon=10^{-20}$

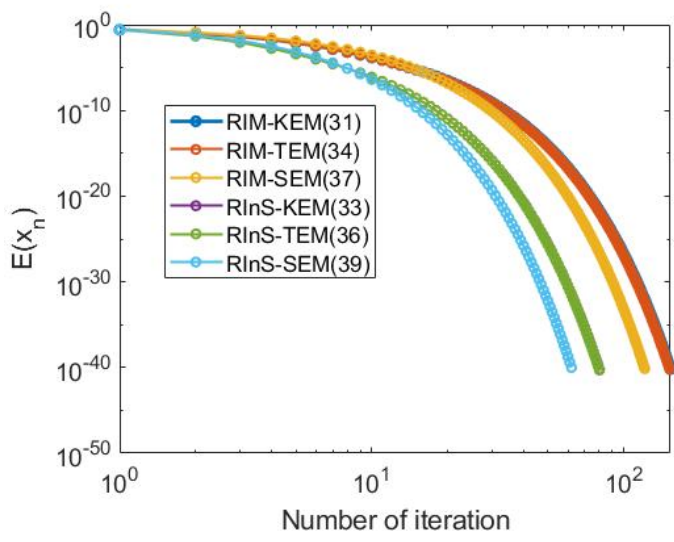

(b) when $\epsilon=10^{-40}$

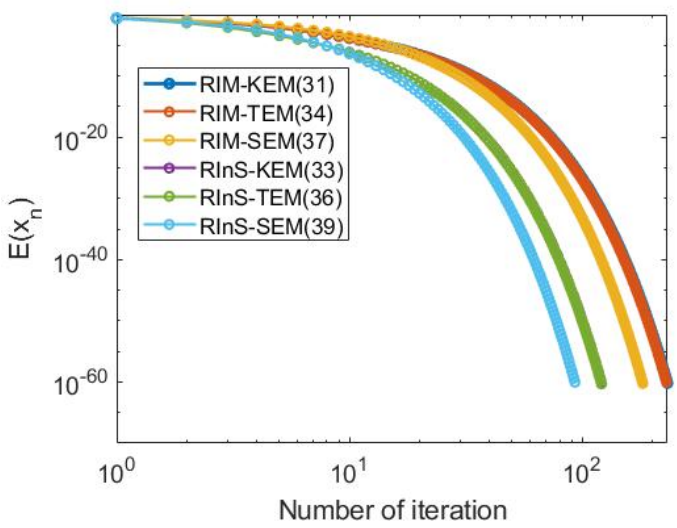

(c) when $\epsilon=10^{-60}$

Fig. 3: Example 5.1: Convergence behaviour of algorithms RIM-KEM(31), RInS-KEM(33), RIMKEM(34), RInS-TEM(36), RIM-SEM(37) and RInS-SEM(39) when $\epsilon=10^{-10}, 10^{-15}, 10^{-20}$

\subsection{Numerical experiments for Example 5.1}

Choose $r=1, s=-1, t=1$ in Example 5.1. Then the operator $F$ defined by (45) is monotone and $L$-Lipschitz continuous on $\mathbb{R}^{3}$, where $L=\sqrt{18}$. It is easy to verify that the point $x^{*}=(0,0,0) \in \mathbb{R}^{3}$ is a solution of problem $\mathrm{VI}(C, F)$. 


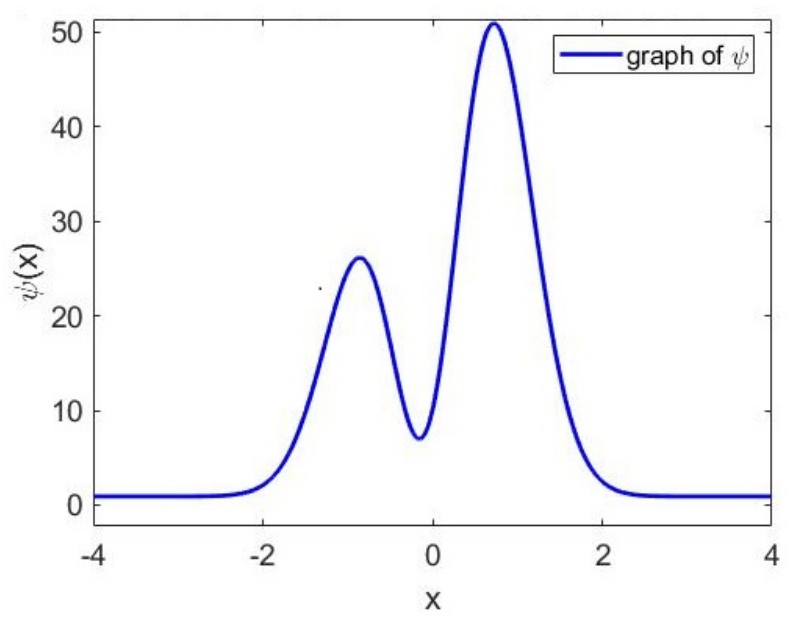

Fig. 4: The graph of $\psi$ defined in Example 5.2 over $[-4,4]$

The numerical experiments are performed for the solution $x^{*}$ by running 500 iterations and using stoping criterion (48) with $\epsilon=10^{-20}, 10^{-40}, 10^{-60}$. In Table 1, we display the iteration history in terms of number of iteration $(n)$, CPU time and $E\left(x_{n}\right)$ for different stopping criterions. The graphical convergence behaviour are reported in Figures 3(a), 3(b), and 3(c) for $\epsilon=10^{-20}, 10^{-40}, 10^{-60}$, respectively. Table 1 indicates that algorithms RInS-KEM(33), RInSTEM(36) and RInS-SEM(39) take significantly less number of iterations and CPU time compared to RIM-KEM(31), RIM-KEM(34) and RIM-SEM(37).

\begin{tabular}{|c|c|c|c|c|c|c|c|c|c|}
\cline { 2 - 11 } \multicolumn{1}{c|}{} & \multicolumn{3}{c|}{$\epsilon=10^{-20}$} & \multicolumn{3}{c|}{$\epsilon=10^{-40}$} & \multicolumn{3}{c|}{$\epsilon=10^{-60}$} \\
\hline Algorithms & Iter. & Time & $E(n)$ & Iter. & Time & $E(n)$ & Iter. & Time & $E(n)$ \\
\hline RIM-KEM(31) & 74 & 0.008 & $6.253 \mathrm{e}-21$ & 153 & 0.003 & $6.527 \mathrm{e}-41$ & 232 & 0.003 & $6.813 \mathrm{e}-61$ \\
\hline RIM-TEM(34) & 73 & 0.002 & $8.499 \mathrm{e}-21$ & 152 & 0.003 & $6.556 \mathrm{e}-41$ & 230 & 0.004 & $9.088 \mathrm{e}-61$ \\
\hline RIM-SEM(37) & 60 & 0.006 & $9.534 \mathrm{e}-21$ & 121 & 0.004 & $7.397 \mathrm{e}-41$ & 182 & 0.005 & $5.739 \mathrm{e}-61$ \\
\hline RInS-KEM(33) & 39 & 0.004 & $5.683 \mathrm{e}-21$ & 80 & 0.003 & $6.144 \mathrm{e}-41$ & 121 & 0.002 & $6.644 \mathrm{e}-61$ \\
\hline RInS-TEM(36) & 39 & 0.001 & $5.141 \mathrm{e}-21$ & 80 & 0.003 & $4.989 \mathrm{e}-41$ & 121 & 0.004 & $4.841 \mathrm{e}-61$ \\
\hline RInS-SEM(39) & 32 & 0.002 & $2.464 \mathrm{e}-21$ & 62 & 0.004 & $9.996 \mathrm{e}-41$ & 93 & 0.003 & $9.153 \mathrm{e}-61$ \\
\hline
\end{tabular}

Table 1: Comparison of RIM-KEM(31), RInS-KEM(33), RIM-KEM(34), RInS-TEM(36), RIM-SEM(37) and RInS$\operatorname{SEM}(39)$ for Example 5.1

\subsection{Numerical experiments for Example 5.2}

For the numerical experiments, in Example 5.2, we take $X=\mathbb{R}^{5}, q=(-1,2,1,0,-1), \alpha=0.1$,

$$
U=\left(\begin{array}{ccccc}
1.5 & 0 & 0 & 0 & 0 \\
0 & 3 & 0 & 0 & 0 \\
0 & 0 & 4.5 & 0 & 0 \\
0 & 0 & 0 & 6 & 0 \\
0 & 0 & 0 & 0 & 7.5
\end{array}\right), V=\left(\begin{array}{ccccc}
5 & -1 & 2 & 0 & 2 \\
-1 & 6 & -1 & 3 & 0 \\
-2 & -1 & -3 & 0 & -1 \\
0 & 3 & 0 & 5 & 0 \\
-2 & 0 & 1 & 0 & 4
\end{array}\right)
$$

Define $\psi:[0, \infty) \rightarrow[0, \infty)$ by

$$
\psi(t)=2\|U\| e^{-\eta t^{2}}\left(\|V\| t^{2}+\|q\| t\right)+\left(e^{-\eta t^{2}}+\alpha\right)\|V\| \text { for all } t \geq 0
$$




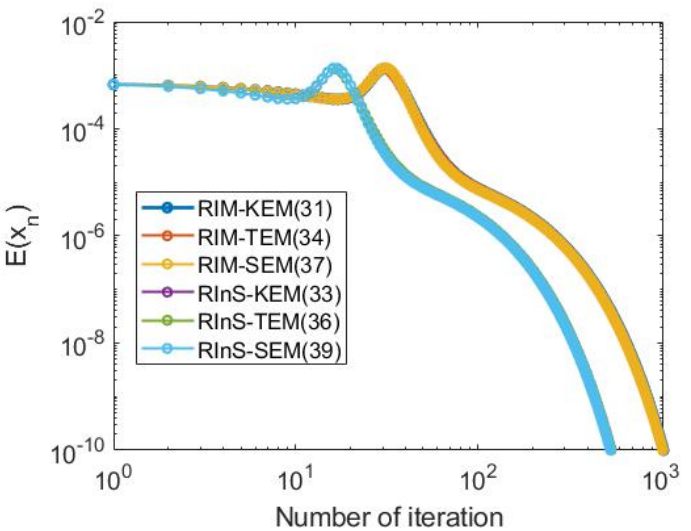

(a) when $\epsilon=10^{-10}$

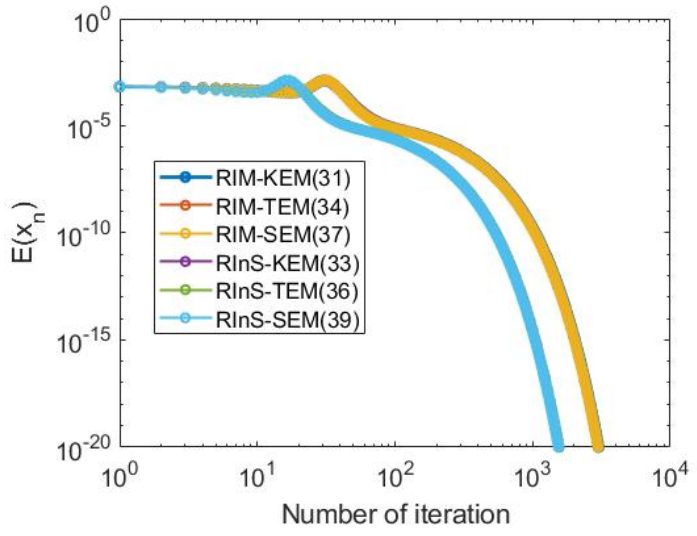

(b) when $\epsilon=10^{-20}$

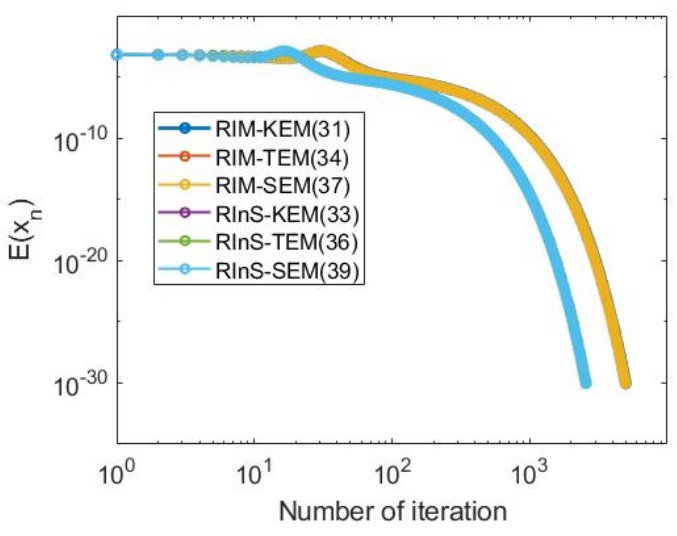

(c) when $\epsilon=10^{-30}$

Fig. 5: Example 5.2: Convergence behaviour of algorithms RIM-KEM(31), RInS-KEM(33), RIM$\operatorname{KEM}(34), \operatorname{RInS}-\mathrm{TEM}(36), \operatorname{RIM-SEM}(37)$ and $\operatorname{RInS}$-SEM(39) when $\epsilon=10^{-10}, 10^{-15}, 10^{-20}$

From Figure 4, we see that $\psi(t)<51$ for all $t \geq 0$. Hence, from (47), we see that $\|\nabla F(x)\| \leq$ $\psi(\|x\|)<51$ for all $x \in X$. This shows that $F$ is 51-Lipschitz continuous on $X$. We compared algorithms RIM-KEM(31), RInS-KEM(33), RIM-KEM(34), RInS-TEM(36), RIM-SEM(37) and RInS$\operatorname{SEM}(39)$ by running 5,000 iterations and using stoping criterion (48) with $\epsilon=10^{-10}, 10^{-20}, 10^{-30}$ for solution of problem $\operatorname{VI}(C, F)$, where the operator $F$ is defined by (46). The graphical convergence behaviour are reported in Figures $5(\mathrm{a}), 5(\mathrm{~b})$ and $5(\mathrm{c})$ for $\epsilon=10^{-10}, 10^{-20}, 10^{-30}$, respectively. From the Table 2, that algorithms RInS-KEM(33), RInS-TEM(36) and RInS-SEM(39) take significantly less number of iterations and CPU time compared to RIM-KEM(31), RIM-KEM(34) and RIM-SEM(37).

Remark 5.1 (i) In Bot et al. [9, Figure 4], it is shown that the performance of the relaxed extragradient method better than subgradient-extragradient method (8). Indeed, Theorems 4.4 and 4.6 are inertial variants of Bot et al. [9, Theorem 3.1].

(ii) In view of Remark 4.6 and Figures 5(a), 5(b) and 5(c) and Tables 2, we report that RInS-KEM(33) and RInS-TEM(36) have better performance compared to RIM-KEM(31) and RIM-TEM(34). 


\begin{tabular}{|c|c|c|c|c|c|c|c|c|c|}
\cline { 2 - 11 } \multicolumn{1}{c|}{} & \multicolumn{3}{c|}{$\epsilon=10^{-10}$} & \multicolumn{3}{c|}{$\epsilon=10^{-20}$} & \multicolumn{3}{c|}{$\epsilon=10^{-30}$} \\
\hline Algorithms & Iter. & Time & $E(n)$ & Iter. & Time & $E(n)$ & Iter. & Time & $E(n)$ \\
\hline RIM-KEM(31) & 1042 & 0.033 & $9.889 \mathrm{e}-11$ & 3005 & 0.036 & $9.911 \mathrm{e}-21$ & 4973 & 0.056 & $9.029 \mathrm{e}-31$ \\
\hline RIM-TEM(34) & 1036 & 0.020 & $9.903 \mathrm{e}-11$ & 2988 & 0.039 & $9.917 \mathrm{e}-21$ & 4944 & 0.053 & $8.998 \mathrm{e}-31$ \\
\hline RIM-SEM(37) & 1034 & 0.033 & $9.960 \mathrm{e}-11$ & 2986 & 0.036 & $9.986 \mathrm{e}-21$ & 4943 & 0.056 & $8.998 \mathrm{e}-31$ \\
\hline RInS-KEM(33) & 539 & 0.023 & $9.893 \mathrm{e}-11$ & 1554 & 0.035 & $9.807 \mathrm{e}-21$ & 2568 & 0.052 & $8.998 \mathrm{e}-31$ \\
\hline RInS-TEM(36) & 538 & 0.021 & $9.859 \mathrm{e}-11$ & 1550 & 0.033 & $9.959 \mathrm{e}-21$ & 2562 & 0.055 & $8.998 \mathrm{e}-31$ \\
\hline RInS-SEM(39) & 534 & 0.033 & $9.901 \mathrm{e}-11$ & 1541 & 0.036 & $9.853 \mathrm{e}-21$ & 2548 & 0.056 & $8.998 \mathrm{e}-31$ \\
\hline
\end{tabular}

Table 2: Comparison of RIM-KEM(31), RInS-KEM(33), RIM-KEM(34), RInS-TEM(36), RIM-SEM(37) and RInS$\operatorname{SEM}(39)$ for Example 5.2

\subsection{Numerical experiments for Example 5.3}

For numerical experiments of RIM-KEM(31), RInS-KEM(33), RIM-KEM(34), RInS-TEM(36), RIM-SEM(37) and RInS-SEM(39), in Example 5.3, we chose three different dimensions $m=$ 520, 1020, 2050.

When $\mathrm{m}=520$ :

We computed the unique solution of the variational inequality $\operatorname{VI}(F, C)$ by running 40,000 iterations and using stoping criterion (48) with $\epsilon=10^{-7}, 10^{-10}, 10^{-15}$. The numerical results are listed in Table 3.

When $\mathbf{m}=1020$ :

We computed the unique solution of the variational inequality $\mathrm{VI}(F, C)$ by running 15,000 iterations and using stoping criterion (48) with $\epsilon=10^{-7}, 10^{-9}, 10^{-11}$. The numerical results are given in Table 4.

When $\mathbf{m}=2050$ :

We computed the unique solution of the variational inequality $\operatorname{VI}(F, C)$ by running 6,000 iterations and using stoping criterion (48) with $\epsilon=10^{-5}, 10^{-7}, 10^{-10}$. The numerical results are listed in Table 5 .

\begin{tabular}{|c|c|c|c|c|c|c|c|c|c|}
\cline { 2 - 11 } \multicolumn{1}{c|}{} & \multicolumn{3}{c|}{$\epsilon=10^{-7}$} & \multicolumn{3}{c|}{$\epsilon=10^{-10}$} & \multicolumn{3}{c|}{$\epsilon=10^{-15}$} \\
\hline Algorithms & Iter. & Time & $E(n)$ & Iter. & Time & $E(n)$ & Iter. & Time & $E(n)$ \\
\hline RIM-KEM(31) & 287 & 0.082 & $9.984 \mathrm{e}-08$ & 5770 & 1.046 & $9.999 \mathrm{e}-11$ & 35583 & 5.931 & $1.000 \mathrm{e}-15$ \\
\hline RIM-TEM(34) & 286 & 0.081 & $9.928 \mathrm{e}-08$ & 5738 & 0.945 & $9.997 \mathrm{e}-11$ & 35383 & 5.796 & $9.999 \mathrm{e}-16$ \\
\hline RIM-SEM(37) & 286 & 0.125 & $9.920 \mathrm{e}-08$ & 5769 & 2.090 & $9.996 \mathrm{e}-11$ & 35578 & 13.040 & $9.997 \mathrm{e}-16$ \\
\hline RInS-KEM(33) & 149 & 0.088 & $9.951 \mathrm{e}-08$ & 2983 & 1.361 & $9.992 \mathrm{e}-11$ & 18389 & 8.252 & $9.998 \mathrm{e}-16$ \\
\hline RInS-TEM(36) & 149 & 0.091 & $9.896 \mathrm{e}-08$ & 2976 & 1.408 & $9.998 \mathrm{e}-11$ & 18350 & 8.010 & $9.996 \mathrm{e}-16$ \\
\hline RInS-SEM(39) & 148 & 0.082 & $9.916 \mathrm{e}-08$ & 2975 & 1.046 & $1.000 \mathrm{e}-10$ & 18347 & 5.931 & $9.996 \mathrm{e}-16$ \\
\hline
\end{tabular}

Table 3: Comparison of RIM-KEM(31), RInS-KEM(33), RIM-KEM(34), RInS-TEM(36), RIM-SEM(37) and RInS$\operatorname{SEM}(39)$ for Example 5.3 with $m=520$

\begin{tabular}{|c|c|c|c|c|c|c|c|c|c|}
\cline { 2 - 11 } \multicolumn{1}{c|}{} & \multicolumn{3}{c|}{$\epsilon=10^{-7}$} & \multicolumn{3}{c|}{$\epsilon=10^{-9}$} & \multicolumn{3}{c|}{$\epsilon=10^{-11}$} \\
\hline Algorithms & Iter. & Time & $E(n)$ & Iter. & Time & $E(n)$ & Iter. & Time & $E(n)$ \\
\hline RIM-KEM(31) & 313 & 0.441 & $9.996 \mathrm{e}-08$ & 1785 & 2.238 & $9.993 \mathrm{e}-10$ & 11900 & 14.786 & $9.998 \mathrm{e}-12$ \\
\hline RIM-TEM(34) & 312 & 0.428 & $9.938 \mathrm{e}-08$ & 1775 & 2.222 & $9.992 \mathrm{e}-10$ & 11833 & 14.678 & $9.998 \mathrm{e}-12$ \\
\hline RIM-SEM(37) & 312 & 0.964 & $9.958 \mathrm{e}-08$ & 1783 & 5.113 & $9.996 \mathrm{e}-10$ & 11898 & 33.424 & $9.999 \mathrm{e}-12$ \\
\hline RInS-KEM(33) & 163 & 0.648 & $9.886 \mathrm{e}-08$ & 923 & 3.330 & $9.991 \mathrm{e}-10$ & 6150 & 21.970 & $9.998 \mathrm{e}-12$ \\
\hline RInS-TEM(36) & 162 & 0.779 & $9.981 \mathrm{e}-08$ & 921 & 3.334 & $9.991 \mathrm{e}-10$ & 6137 & 21.980 & $9.998 \mathrm{e}-12$ \\
\hline RInS-SEM(39) & 162 & 0.441 & $9.868 \mathrm{e}-08$ & 920 & 2.238 & $9.994 \mathrm{e}-10$ & 6136 & 14.786 & $9.999 \mathrm{e}-12$ \\
\hline
\end{tabular}

Table 4: Comparison of RIM-KEM(31), RInS-KEM(33), RIM-KEM(34), RInS-TEM(36), RIM-SEM(37) and RInS$\operatorname{SEM}(39)$ for Example 5.3 with $m=1020$ 


\begin{tabular}{|c|c|c|c|c|c|c|c|c|c|}
\cline { 2 - 11 } \multicolumn{1}{c|}{} & \multicolumn{3}{c|}{$\epsilon=10^{-20}$} & \multicolumn{3}{c|}{$\epsilon=10^{-40}$} & \multicolumn{3}{c|}{$\epsilon=10^{-60}$} \\
\hline Algorithms & Iter. & Time & $f\left(x_{n}\right)$ & Iter. & Time & $f\left(x_{n}\right)$ & Iter. & Time & $f\left(x_{n}\right)$ \\
\hline RIM-KEM(31) & 99 & 0.007 & $7.230 \mathrm{e}-21$ & 199 & 0.004 & $8.146 \mathrm{e}-41$ & 299 & 0.005 & $9.177 \mathrm{e}-61$ \\
\hline RIM-TEM(34) & 98 & 0.004 & $8.611 \mathrm{e}-21$ & 198 & 0.006 & $7.243 \mathrm{e}-41$ & 297 & 0.009 & $9.671 \mathrm{e}-61$ \\
\hline RIM-SEM(37) & 20 & 0.006 & $5.372 \mathrm{e}-21$ & 36 & 0.002 & $1.248 \mathrm{e}-41$ & 51 & 0.002 & $5.651 \mathrm{e}-61$ \\
\hline RInS-KEM(33) & 52 & 0.004 & $6.321 \mathrm{e}-21$ & 104 & 0.006 & $6.400 \mathrm{e}-41$ & 156 & 0.008 & $6.479 \mathrm{e}-61$ \\
\hline RInS-TEM(36) & 52 & 0.002 & $5.696 \mathrm{e}-21$ & 104 & 0.009 & $5.186 \mathrm{e}-41$ & 156 & 0.009 & $4.721 \mathrm{e}-61$ \\
\hline RInS-SEM(39) & 11 & 0.001 & $8.639 \mathrm{e}-21$ & 20 & 0.002 & $1.831 \mathrm{e}-42$ & 28 & 0.002 & $9.932 \mathrm{e}-62$ \\
\hline
\end{tabular}

Table 6: Example 4.1 with $d=0.8$

\begin{tabular}{|c|c|c|c|c|c|c|c|c|c|}
\cline { 2 - 11 } \multicolumn{1}{c|}{} & \multicolumn{3}{c|}{$\epsilon=10^{-20}$} & \multicolumn{3}{c|}{$\epsilon=10^{-40}$} & \multicolumn{3}{c|}{$\epsilon=10^{-60}$} \\
\hline Algorithms & Iter. & Time & $f\left(x_{n}\right)$ & Iter. & Time & $f\left(x_{n}\right)$ & Iter. & Time & $f\left(x_{n}\right)$ \\
\hline RIM-KEM(31) & 99 & 0.008 & $6.827 \mathrm{e}-21$ & 199 & 0.006 & $7.691 \mathrm{e}-41$ & 299 & 0.005 & $8.665 \mathrm{e}-61$ \\
\hline RIM-TEM(34) & 98 & 0.002 & $8.128 \mathrm{e}-21$ & 198 & 0.006 & $6.836 \mathrm{e}-41$ & 297 & 0.011 & $9.128 \mathrm{e}-61$ \\
\hline RIM-SEM(37) & 20 & 0.007 & $8.157 \mathrm{e}-22$ & 35 & 0.003 & $3.693 \mathrm{e}-41$ & 51 & 0.003 & $8.580 \mathrm{e}-62$ \\
\hline RInS-KEM(33) & 52 & 0.005 & $5.975 \mathrm{e}-21$ & 104 & 0.006 & $6.049 \mathrm{e}-41$ & 156 & 0.013 & $6.124 \mathrm{e}-61$ \\
\hline RInS-TEM(36) & 52 & 0.002 & $5.383 \mathrm{e}-21$ & 104 & 0.008 & $4.900 \mathrm{e}-41$ & 156 & 0.008 & $4.461 \mathrm{e}-61$ \\
\hline RInS-SEM(39) & 11 & 0.001 & $1.415 \mathrm{e}-21$ & 19 & 0.002 & $7.674 \mathrm{e}-41$ & 28 & 0.002 & $1.626 \mathrm{e}-62$ \\
\hline
\end{tabular}

Table 7: Example 4.1 with $d=1$

\begin{tabular}{|c|c|c|c|c|c|c|c|c|c|}
\cline { 2 - 11 } \multicolumn{1}{c|}{} & \multicolumn{3}{c|}{$\epsilon=10^{-20}$} & \multicolumn{3}{c|}{$\epsilon=10^{-40}$} & \multicolumn{3}{c|}{$\epsilon=10^{-60}$} \\
\hline Algorithms & Iter. & Time & $f\left(x_{n}\right)$ & Iter. & Time & $f\left(x_{n}\right)$ & Iter. & Time & $f\left(x_{n}\right)$ \\
\hline RIM-KEM(31) & 99 & 0.008 & $6.801 \mathrm{e}-21$ & 199 & 0.006 & $7.662 \mathrm{e}-41$ & 299 & 0.006 & $8.633 \mathrm{e}-61$ \\
\hline RIM-TEM(34) & 98 & 0.002 & $8.096 \mathrm{e}-21$ & 198 & 0.013 & $6.809 \mathrm{e}-41$ & 297 & 0.009 & $9.092 \mathrm{e}-61$ \\
\hline RIM-SEM(37) & 19 & 0.007 & $4.678 \mathrm{e}-21$ & 35 & 0.003 & $1.087 \mathrm{e}-41$ & 50 & 0.005 & $4.921 \mathrm{e}-61$ \\
\hline RInS-KEM(33) & 52 & 0.004 & $5.961 \mathrm{e}-21$ & 104 & 0.005 & $6.035 \mathrm{e}-41$ & 156 & 0.008 & $6.110 \mathrm{e}-61$ \\
\hline RInS-TEM(36) & 52 & 0.003 & $5.370 \mathrm{e}-21$ & 104 & 0.007 & $4.889 \mathrm{e}-41$ & 156 & 0.010 & $4.450 \mathrm{e}-61$ \\
\hline RInS-SEM(39) & 11 & 0.001 & $4.416 \mathrm{e}-22$ & 19 & 0.004 & $2.395 \mathrm{e}-41$ & 28 & 0.003 & $5.077 \mathrm{e}-63$ \\
\hline
\end{tabular}

Table 8: Example 4.1 with $d=1.2$

\subsection{Numerical experiments for pseudo-convex optimization problem (43) with Example 4.1}

Consider pseudo-convex optimization problem (43), where the objective function $f$ is defined by (44) (see Example 4.1). Note that $F=\nabla f$ is pseudo-monotone and $\frac{125}{54 d}$-Lipschitz continuous on $X=\mathbb{R}^{m}$. Here we take $m=500$ and $d=0.8,1,1.2$.

For numerical experiments of RIM-KEM(31), RInS-KEM(33), RIM-KEM(34), RInS-TEM(36), RIM-SEM(37) and RInS-SEM(39), we chose three different $d=0.8,1,1.2$. We computed the minimizer of pseudo-convex optimization problem (43) by running 500 iterations and using stoping criterion $f\left(x_{n}\right) \leq \epsilon$ for $\epsilon=10^{-20}, 10^{-40}, 10^{-60}$ and the corresponding numerical results are listed in Tables 6,7 and 8 , respectively. 


\section{Concluding remarks and further research}

In this paper, we have investigated a unified framework for well known three extragradient methods $\operatorname{KEM}(4), \operatorname{TEM}(7)$ and $\operatorname{SEM}(8)$ and investigated computational theory of the inertial extragradient methods with relaxation parameters for solving infinite-dimensional variational inequality problem over a nonempty convex closed set governed by a pseudo-monotone and Lipschitz continuous operator. Indeed, we investigated that the inertial fixed point iterative methods with relaxation parameters can be transformed into the inertial extragradient methods for efficiently solving variational inequality and pseudo-convex optimization problems. Our operator theoretic approach provides affirmative answer $(\mathbf{Q 1})$ and $(\mathbf{Q} 2)$. Numerical experiments show that the relaxed inertial normal S-iterative technique has a better convergence behavior compared to the relaxed inertial Mann iterative variant of Tseng's method, Korpelevich's extragradient method and also the subgradient-extragradient method. The strong convergence of the extragradient methods with relaxation parameters is still an open question that could be an interesting topic for a future research.

\section{Appendix-I: Proof of Lemma 2.5}

Proof (a) Set $K:=\varepsilon-\theta(1+\varepsilon+\max \{1, \varepsilon\})$. Define $K_{n}=\theta_{n}\left[1+\theta_{n}+\varepsilon\left(1-\theta_{n}\right)\right], n=1,2, \cdots$ and $\phi_{n}=\left\|x_{n}-v\right\|^{2}, n=0,1,2, \cdots$. From (11), we have

$$
\phi_{n+1} \leq\left(1+\theta_{n}\right) \phi_{n}-\theta_{n} \phi_{n-1}-\varepsilon\left(1-\theta_{n}\right)\left\|x_{n+1}-x_{n}\right\|^{2}+K_{n}\left\|x_{n}-x_{n-1}\right\|^{2} \text { for all } n \in \mathbb{N}
$$

and hence

$$
\phi_{n+1}-\left(1+\theta_{n}\right) \phi_{n}+\theta_{n} \phi_{n-1} \leq-\varepsilon\left(1-\theta_{n}\right)\left\|x_{n+1}-x_{n}\right\|^{2}+K_{n}\left\|x_{n}-x_{n-1}\right\|^{2} \text { for all } n \in \mathbb{N} .
$$

Now

$$
\begin{aligned}
\varphi_{n+1}-\varphi_{n} & =\phi_{n+1}-\theta_{n+1} \phi_{n}+K_{n+1}\left\|x_{n+1}-x_{n}\right\|^{2}-\left(\phi_{n}-\theta_{n} \phi_{n-1}+K_{n}\left\|x_{n}-x_{n-1}\right\|^{2}\right) \\
& =\phi_{n+1}-\left(1+\theta_{n+1}\right) \phi_{n}+\theta_{n} \phi_{n-1}+K_{n+1}\left\|x_{n+1}-x_{n}\right\|^{2}-K_{n}\left\|x_{n}-x_{n-1}\right\|^{2} \\
& \leq-\varepsilon\left(1-\theta_{n}\right)\left\|x_{n+1}-x_{n}\right\|^{2}+K_{n}\left\|x_{n}-x_{n-1}\right\|^{2}+K_{n+1}\left\|x_{n+1}-x_{n}\right\|^{2}-K_{n}\left\|x_{n}-x_{n-1}\right\|^{2} \\
& =-\left[\varepsilon\left(1-\theta_{n}\right)-K_{n+1}\right]\left\|x_{n+1}-x_{n}\right\|^{2} .
\end{aligned}
$$

Note $\theta_{n} \leq \theta_{n+1} \leq \theta$ for all $n \in \mathbb{N}$ and

$$
K_{n}=\theta_{n}\left(1+\theta_{n}+\varepsilon\left(1-\theta_{n}\right)\right) \leq \theta_{n}(1+\max \{1, \varepsilon\}) \text { for all } n \in \mathbb{N} .
$$

Hence

$$
\begin{aligned}
-\left[\varepsilon\left(1-\theta_{n}\right)-K_{n+1}\right] & =-\varepsilon\left(1-\theta_{n}\right)+\theta_{n+1}\left(1+\theta_{n+1}+\varepsilon\left(1-\theta_{n+1}\right)\right) \\
& \leq-\varepsilon\left(1-\theta_{n}\right)+\theta_{n+1}(1+\max \{1, \varepsilon\}) \\
& \leq-\varepsilon(1-\theta)+\theta(1+\max \{1, \varepsilon\}) \\
& =-[\varepsilon-\theta(1+\varepsilon+\max \{1, \varepsilon\})]=-K .
\end{aligned}
$$

Note $K>0$ by the inequality (12). Hence

$$
\varphi_{n+1}-\varphi_{n} \leq-K\left\|x_{n+1}-x_{n}\right\|^{2}, \quad n \in \mathbb{N},
$$

which implies that $\varphi_{n+1} \leq \varphi_{n}$ for all $n \in \mathbb{N}$. Thus, the sequence $\left\{\varphi_{n}\right\}$ is non-increasing.

(b) For $n \in \mathbb{N}$, we have

$$
\varphi_{n}=\phi_{n}-\theta_{n} \phi_{n-1}+K_{n}\left\|x_{n}-x_{n-1}\right\|^{2} \geq \phi_{n}-\theta_{n} \phi_{n-1}
$$

and

$$
\varphi_{n+1}=\phi_{n+1}-\theta_{n+1} \phi_{n}+K_{n+1}\left\|x_{n+1}-x_{n}\right\|^{2} \geq-\theta_{n+1} \phi_{n} .
$$


Since $\varphi_{1}>0$, from (51), we have

$$
\begin{aligned}
\phi_{n} & \leq \theta_{n} \phi_{n-1}+\varphi_{n} \\
& \leq \theta \phi_{n-1}+\varphi_{1} \\
& \cdots \\
& \leq \theta^{n} \phi_{0}+\left(1+\theta+\theta^{2}+\cdots+\theta^{n-1}\right) \varphi_{1} \\
& \leq \theta^{n} \phi_{0}+\frac{\varphi_{1}}{1-\theta}, \quad n \in \mathbb{N} .
\end{aligned}
$$

Combining (52) and (53), we have

$$
-\varphi_{n+1} \leq \theta_{n+1} \phi_{n} \leq \theta \phi_{n} \leq \theta^{n+1} \phi_{0}+\frac{\theta \varphi_{1}}{1-\theta} .
$$

From (50), we get

$$
K \sum_{i=1}^{n}\left\|x_{i+1}-x_{i}\right\|^{2} \leq \varphi_{1}-\varphi_{n+1} \leq \varphi_{1}+\theta^{n+1} \phi_{0}+\frac{\theta \varphi_{1}}{1-\theta} \text { for all } n \in \mathbb{N} .
$$

Taking limit as $n \rightarrow \infty$, we have

$$
K \sum_{i=1}^{\infty}\left\|x_{i+1}-x_{i}\right\|^{2} \leq \frac{\varphi_{1}}{1-\theta}<\infty
$$

(c) From (49), we have

$$
\phi_{n+1} \leq\left(1+\theta_{n}\right) \phi_{n}-\theta_{n} \phi_{n-1}+\theta(1+\max \{1, \varepsilon\})\left\|x_{n}-x_{n-1}\right\|^{2} .
$$

From Lemma 2.4, we obtain that $\lim _{n \rightarrow \infty}\left\|x_{n}-v\right\|$ exists.

\section{Appendix-II: Proof of Proposition 3.1}

Proof (a)-(b). Let $v \in \operatorname{Fix}(S)$. Note $T w_{n}-w_{n}=\frac{1}{\alpha_{n}}\left(x_{n+1}-w_{n}\right)$ and $\varepsilon \leq \frac{1+\kappa-\alpha_{n}}{\alpha_{n}}$ for all $n \in \mathbb{N}$. Note $T$ is $\kappa$-strongly quasi-nonexpansive operator. From (13) and Lemma 2.2(ii), we have

$$
\begin{aligned}
\left\|x_{n+1}-v\right\|^{2} & =\left\|\left(1-\alpha_{n}\right) w_{n}+\alpha_{n} T w_{n}-v\right\|^{2} \\
& =\left(1-\alpha_{n}\right)\left\|w_{n}-v\right\|^{2}+\alpha_{n}\left\|T w_{n}-v\right\|^{2}-\alpha_{n}\left(1-\alpha_{n}\right)\left\|w_{n}-T w_{n}\right\|^{2} \\
& \leq\left(1-\alpha_{n}\right)\left\|w_{n}-v\right\|^{2}+\alpha_{n}\left(\left\|w_{n}-v\right\|^{2}-\kappa\left\|w_{n}-T w_{n}\right\|^{2}\right)-\alpha_{n}\left(1-\alpha_{n}\right)\left\|w_{n}-T w_{n}\right\|^{2} \\
& =\left\|w_{n}-v\right\|^{2}-\alpha_{n}\left(1+\kappa-\alpha_{n}\right)\left\|w_{n}-T w_{n}\right\|^{2} \\
& =\left\|w_{n}-v\right\|^{2}-\frac{1+\kappa-\alpha_{n}}{\alpha_{n}}\left\|x_{n+1}-w_{n}\right\|^{2} .
\end{aligned}
$$

Again, from (13) and Lemma 2.2(ii), we have

$$
\begin{aligned}
\left\|w_{n}-v\right\|^{2} & =\left\|\left(1+\theta_{n}\right)\left(x_{n}-v\right)-\theta_{n}\left(x_{n-1}-v\right)\right\|^{2} \\
& =\left(1+\theta_{n}\right)\left\|x_{n}-v\right\|^{2}-\theta_{n}\left\|x_{n-1}-v\right\|^{2}+\theta_{n}\left(1+\theta_{n}\right)\left\|x_{n}-x_{n-1}\right\|^{2} .
\end{aligned}
$$

Combining (55) and (56), we get

$$
\begin{aligned}
\left\|x_{n+1}-v\right\|^{2} \leq & \left(1+\theta_{n}\right)\left\|x_{n}-v\right\|^{2}-\theta_{n}\left\|x_{n-1}-v\right\|^{2}+\theta_{n}\left(1+\theta_{n}\right)\left\|x_{n}-x_{n-1}\right\|^{2} \\
& -\varepsilon\left\|x_{n+1}-w_{n}\right\|^{2} .
\end{aligned}
$$

From (10), we have

$$
\left\|x_{n+1}-w_{n}\right\|^{2}=\left\|x_{n+1}-x_{n}-\theta_{n}\left(x_{n}-x_{n-1}\right)\right\|^{2} \geq\left(1-\theta_{n}\right)\left\|x_{n+1}-x_{n}\right\|^{2}-\theta_{n}\left(1-\theta_{n}\right)\left\|x_{n}-x_{n-1}\right\|^{2} .
$$


From (57), we obtain

$$
\begin{aligned}
\left\|x_{n+1}-v\right\|^{2} \leq & \left(1+\theta_{n}\right)\left\|x_{n}-v\right\|^{2}-\theta_{n}\left\|x_{n-1}-v\right\|^{2}+\theta_{n}\left(1+\theta_{n}\right)\left\|x_{n}-x_{n-1}\right\|^{2}-\varepsilon\left(1-\theta_{n}\right)\left\|x_{n+1}-x_{n}\right\|^{2} \\
& +\varepsilon \theta_{n}\left(1-\theta_{n}\right)\left\|x_{n}-x_{n-1}\right\|^{2} \\
= & \left(1+\theta_{n}\right)\left\|x_{n}-v\right\|^{2}-\theta_{n}\left\|x_{n-1}-v\right\|^{2}-\varepsilon\left(1-\theta_{n}\right)\left\|x_{n+1}-x_{n}\right\|^{2} \\
& +\theta_{n}\left(1+\theta_{n}+\varepsilon\left(1-\theta_{n}\right)\right)\left\|x_{n}-x_{n-1}\right\|^{2} .
\end{aligned}
$$

Noticing that $\left\{\theta_{n}\right\}$ is an increasing sequence in $[0,1)$ by the assumption $(\mathbf{C} 2)$ and that the condition (12) holds by the assumption (C3). Apply Lemma 2.5 on (58), we conclude that $\sum_{n=1}^{\infty} \| x_{n+1}-$ $x_{n} \|^{2}<\infty$ and $\lim _{n \rightarrow \infty}\left\|x_{n}-v\right\|$ exists.

Clearly, $\left\|x_{n+1}-x_{n}\right\| \rightarrow 0$ as $n \rightarrow \infty$. Hence, from (13), we have

$$
\left\|w_{n}-x_{n+1}\right\| \leq\left\|x_{n}-x_{n+1}\right\|+\theta\left\|x_{n}-x_{n-1}\right\| \rightarrow 0 .
$$

It follows that $\lim _{n \rightarrow \infty}\left\|w_{n}-x_{n}\right\|=0$.

We may assume that $\lim _{n \rightarrow \infty}\left\|x_{n}-v\right\|=\ell>0$. It follows, from $\lim _{n \rightarrow \infty}\left\|x_{n}-w_{n}\right\|=0$, that $\lim _{n \rightarrow \infty}\left\|w_{n}-v\right\|=\ell$. In view of condition (C1), we obtain, from (54), that $\lim _{n \rightarrow \infty}\left\|w_{n}-T w_{n}\right\|=$ 0 .

(c) Let $v \in \operatorname{Fix}(S)$. Note $R_{T}(n):=R_{T,\left\{w_{n}\right\}}(n) \leq\left\|w_{n}-T w_{n}\right\|$ for all $n \in \mathbb{N}$. From (54) and (56), we have

$$
\begin{aligned}
\left\|x_{n+1}-v\right\|^{2} \leq & \left(1+\theta_{n}\right)\left\|x_{n}-v\right\|^{2}-\theta_{n}\left\|x_{n-1}-v\right\|^{2}+\theta_{n}\left(1+\theta_{n}\right)\left\|x_{n}-x_{n-1}\right\|^{2} \\
& -\alpha_{n}\left(1+\kappa-\alpha_{n}\right)\left\|w_{n}-T w_{n}\right\|^{2} \\
\leq & \left\|x_{n}-v\right\|^{2}+\left(\theta_{n+1}\left\|x_{n}-v\right\|^{2}-\theta_{n}\left\|x_{n-1}-v\right\|^{2}\right)+\theta(1+\theta)\left\|x_{n}-x_{n-1}\right\|^{2} \\
& -\alpha_{n}\left(1+\kappa-\alpha_{n}\right)\left\|w_{n}-T w_{n}\right\|^{2} .
\end{aligned}
$$

Hence, from the condition $(\mathbf{C} 1)$, we have

$$
\begin{aligned}
a(1+\kappa-b) \sum_{i=1}^{n}\left\|w_{i}-T w_{i}\right\|^{2} \leq & \sum_{i=1}^{n}\left(\left\|x_{i}-v\right\|^{2}-\left\|x_{i+1}-v\right\|^{2}\right)+\sum_{i=1}^{n}\left(\theta_{i+1}\left\|x_{i}-v\right\|^{2}-\theta_{i}\left\|x_{i-1}-v\right\|^{2}\right) \\
& +\theta(1+\theta) \sum_{i=1}^{n}\left\|x_{i}-x_{i-1}\right\|^{2} \\
= & \left\|x_{1}-v\right\|^{2}-\left\|x_{n+1}-v\right\|^{2}+\theta_{n+1}\left\|x_{n}-v\right\|^{2}-\theta_{1}\left\|x_{0}-v\right\|^{2} \\
& +\theta(1+\theta) \sum_{i=1}^{n}\left\|x_{i}-x_{i-1}\right\|^{2} \\
\leq & \left\|x_{1}-v\right\|^{2}+\theta_{n+1}\left\|x_{n}-v\right\|^{2}+\theta(1+\theta) \sum_{i=1}^{\infty}\left\|x_{i}-x_{i-1}\right\|^{2} \\
\leq & M
\end{aligned}
$$

for all $n \in \mathbb{N}$ and for some $M>0$. Thus, $\sum_{n=1}^{\infty}\left(R_{T}(n)\right)^{2}<\infty$. Note $\left\{R_{T}(n)\right\}$ is decreasing. Hence, from Lemma 2.3, we see that (16) holds.

\section{Appendix-III: Proof of Proposition 3.2}

Proof (a)-(b). Let $v \in \operatorname{Fix}(S)$. Set $y_{n}:=\left(1-\alpha_{n}\right) w_{n}+\alpha_{n} T w_{n}$. Hence $T w_{n}-w_{n}=\frac{1}{\alpha_{n}}\left(y_{n}-w_{n}\right)$.

From (20), we have $\mathcal{E} \leq \frac{\alpha_{n}\left(1+\kappa-\alpha_{n}\right)}{2\left(1+\alpha_{n}^{2} \beta^{2}\right)}$ for all $n \in \mathbb{N}$. For $\rho=\frac{1}{2}$, from (9), we obtain

$$
\left\|x_{n+1}-T w_{n}\right\|^{2}=\left\|x_{n+1}-w_{n}+w_{n}-T w_{n}\right\|^{2} \geq \frac{1}{2}\left\|x_{n+1}-w_{n}\right\|^{2}-\left\|w_{n}-T w_{n}\right\|^{2} .
$$


Since $T$ is $\beta$-Lipschitz continuous, we have

$$
\begin{aligned}
\left\|w_{n}-T w_{n}\right\|^{2}=\frac{1}{\alpha_{n}^{2}}\left\|y_{n}-w_{n}\right\|^{2} & \geq \frac{1}{\alpha_{n}^{2} \beta^{2}}\left\|T y_{n}-T w_{n}\right\|^{2}=\frac{1}{\alpha_{n}^{2} \beta^{2}}\left\|x_{n+1}-T w_{n}\right\|^{2} \\
& \geq \frac{1}{\alpha_{n}^{2} \beta^{2}}\left(\frac{1}{2}\left\|x_{n+1}-w_{n}\right\|^{2}-\left\|w_{n}-T w_{n}\right\|^{2}\right) .
\end{aligned}
$$

Thus,

$$
\left\|w_{n}-T w_{n}\right\|^{2} \geq \frac{1}{2\left(1+\alpha_{n}^{2} \beta^{2}\right)}\left\|x_{n+1}-w_{n}\right\|^{2} .
$$

Note

$$
\begin{aligned}
\left\|y_{n}-v\right\|^{2} & =\left\|\left(1-\alpha_{n}\right) w_{n}+\alpha_{n} T w_{n}-v\right\|^{2} \\
& =\left(1-\alpha_{n}\right)\left\|w_{n}-v\right\|^{2}+\alpha_{n}\left\|T w_{n}-v\right\|^{2}-\alpha_{n}\left(1-\alpha_{n}\right)\left\|w_{n}-T w_{n}\right\|^{2} \\
& \leq\left(1-\alpha_{n}\right)\left\|w_{n}-v\right\|^{2}+\alpha_{n}\left(\left\|w_{n}-v\right\|^{2}-\kappa\left\|w_{n}-T w_{n}\right\|^{2}\right)-\alpha_{n}\left(1-\alpha_{n}\right)\left\|w_{n}-T w_{n}\right\|^{2} \\
& =\left\|w_{n}-v\right\|^{2}-\alpha_{n}\left(1+\kappa-\alpha_{n}\right)\left\|w_{n}-T w_{n}\right\|^{2} .
\end{aligned}
$$

Using (60) and then (59), we obtain

$$
\begin{aligned}
\left\|x_{n+1}-v\right\|^{2} & =\left\|T y_{n}-v\right\|^{2} \\
& \leq\left\|y_{n}-v\right\|^{2}-\kappa\left\|y_{n}-T y_{n}\right\|^{2} \\
& \leq\left\|w_{n}-v\right\|^{2}-\alpha_{n}\left(1+\kappa-\alpha_{n}\right)\left\|w_{n}-T w_{n}\right\|^{2}-\kappa\left\|y_{n}-T y_{n}\right\|^{2} \\
& =\left\|w_{n}-v\right\|^{2}-\frac{\alpha_{n}\left(1+\kappa-\alpha_{n}\right)}{2\left(1+\alpha_{n}^{2} \beta^{2}\right)}\left\|x_{n+1}-w_{n}\right\|^{2}-\kappa\left\|y_{n}-T y_{n}\right\|^{2} \\
& \leq\left\|w_{n}-v\right\|^{2}-\mathcal{E}\left\|x_{n+1}-w_{n}\right\|^{2}-\kappa\left\|y_{n}-T y_{n}\right\|^{2} .
\end{aligned}
$$

From (18) and Lemma 2.2(ii), we have

$$
\begin{aligned}
\left\|w_{n}-v\right\|^{2} & =\left\|x_{n}+\theta_{n}\left(x_{n}-x_{n-1}\right)-v\right\|^{2} \\
& =\left(1+\theta_{n}\right)\left\|x_{n}-v\right\|^{2}-\theta_{n}\left\|x_{n-1}-v\right\|^{2}+\theta_{n}\left(1+\theta_{n}\right)\left\|x_{n}-x_{n-1}\right\|^{2} .
\end{aligned}
$$

From (10), we have

$$
\left\|x_{n+1}-w_{n}\right\|^{2}=\left\|x_{n+1}-x_{n}-\theta_{n}\left(x_{n}-x_{n-1}\right)\right\|^{2} \geq\left(1-\theta_{n}\right)\left\|x_{n+1}-x_{n}\right\|^{2}-\theta_{n}\left(1-\theta_{n}\right)\left\|x_{n}-x_{n-1}\right\|^{2} .
$$

From (62), we obtain

$$
\begin{aligned}
\left\|x_{n+1}-v\right\|^{2} \leq & \left(1+\theta_{n}\right)\left\|x_{n}-v\right\|^{2}-\theta_{n}\left\|x_{n-1}-v\right\|^{2}+\theta_{n}\left(1+\theta_{n}\right)\left\|x_{n}-x_{n-1}\right\|^{2} \\
& -\mathcal{E}\left\|x_{n+1}-w_{n}\right\|^{2}-\kappa\left\|y_{n}-T y_{n}\right\|^{2} \\
\leq & \left(1+\theta_{n}\right)\left\|x_{n}-v\right\|^{2}-\theta_{n}\left\|x_{n-1}-v\right\|^{2}+\theta_{n}\left(1+\theta_{n}\right)\left\|x_{n}-x_{n-1}\right\|^{2}-\mathcal{E}\left(1-\theta_{n}\right)\left\|x_{n+1}-x_{n}\right\|^{2} \\
& +\mathcal{E} \theta_{n}\left(1-\theta_{n}\right)\left\|x_{n}-x_{n-1}\right\|^{2}-\kappa\left\|y_{n}-T y_{n}\right\|^{2} \\
= & \left(1+\theta_{n}\right)\left\|x_{n}-v\right\|^{2}-\theta_{n}\left\|x_{n-1}-v\right\|^{2}-\mathcal{E}\left(1-\theta_{n}\right)\left\|x_{n+1}-x_{n}\right\|^{2} \\
& +\theta_{n}\left(1+\theta_{n}+\mathcal{E}\left(1-\theta_{n}\right)\right)\left\|x_{n}-x_{n-1}\right\|^{2}-\kappa\left\|y_{n}-T y_{n}\right\|^{2} .
\end{aligned}
$$

Note $\left\{\theta_{n}\right\}$ is an increasing sequence in $[0,1)$ by the assumption $(\mathbf{C} 2)$ and the condition (12) holds by the assumption $(\mathbf{C} 5)$ with $\varepsilon=\mathcal{E}$. Thus, Lemma 2.5 infers us that $\sum_{n=1}^{\infty}\left\|x_{n+1}-x_{n}\right\|^{2}<\infty$ and $\lim _{n \rightarrow \infty}\left\|x_{n}-v\right\|$ exists. It immediately follows, from (18), that $\lim _{n \rightarrow \infty}\left\|w_{n}-x_{n}\right\|=0$.

Since $\ell:=\lim _{n \rightarrow \infty}\left\|x_{n}-v\right\|$ exists, it follows, from $\lim _{n \rightarrow \infty}\left\|x_{n}-w_{n}\right\|=0$, that $\lim _{n \rightarrow \infty} \| w_{n}-$ $v \|=\ell$. Hence, from (61), we obtain that $\lim _{n \rightarrow \infty}\left\|w_{n}-T w_{n}\right\|=0=\lim _{n \rightarrow \infty}\left\|y_{n}-T y_{n}\right\|$.

(c) Let $v \in \operatorname{Fix}(S)$. Note $\left\{\theta_{n}\right\}$ is an increasing sequence in $[0,1)$. From (61) and (63), we get

$$
\begin{aligned}
\left\|x_{n+1}-v\right\|^{2} \leq & \left(1+\theta_{n}\right)\left\|x_{n}-v\right\|^{2}-\theta_{n}\left\|x_{n-1}-v\right\|^{2}+\theta_{n}\left(1+\theta_{n}\right)\left\|x_{n}-x_{n-1}\right\|^{2} \\
& -\alpha_{n}\left(1+\kappa-\alpha_{n}\right)\left\|w_{n}-T w_{n}\right\|^{2}-\kappa\left\|y_{n}-T y_{n}\right\|^{2} .
\end{aligned}
$$


Hence, from the condition $(\mathbf{C} 1)$, we have

$$
\begin{aligned}
& a(1+\kappa-b) \sum_{i=1}^{n}\left\|w_{i}-T w_{i}\right\|^{2}+\kappa \sum_{i=1}^{n}\left\|y_{i}-T y_{i}\right\|^{2} \\
\leq & \sum_{i=1}^{n}\left(\left\|x_{i}-v\right\|^{2}-\left\|x_{i+1}-v\right\|^{2}\right)+\sum_{i=1}^{n}\left(\theta_{i+1}\left\|x_{i}-v\right\|^{2}-\theta_{i}\left\|x_{i-1}-v\right\|^{2}\right)+\theta(1+\theta) \sum_{i=1}^{n}\left\|x_{i}-x_{i-1}\right\|^{2} \\
\leq & \left\|x_{1}-v\right\|^{2}-\left\|x_{n+1}-v\right\|^{2}+\theta_{n+1}\left\|x_{n}-v\right\|^{2}-\theta_{1}\left\|x_{0}-v\right\|^{2}+\theta(1+\theta) \sum_{i=1}^{n}\left\|x_{i}-x_{i-1}\right\|^{2} \\
\leq & \left\|x_{1}-v\right\|^{2}+\theta_{n+1}\left\|x_{n}-v\right\|^{2}+\theta(1+\theta) \sum_{i=1}^{\infty}\left\|x_{i}-x_{i-1}\right\|^{2}
\end{aligned}
$$

$\leq M$ for all $n \in \mathbb{N}$ and for some $M>0$.

Since $T$ is $\beta$-Lipschitz continuous, we have

$$
\left\|x_{n+1}-T x_{n+1}\right\|=\left\|T y_{n}-T x_{n+1}\right\| \leq \beta\left\|y_{n}-x_{n+1}\right\|=\beta\left\|y_{n}-T y_{n}\right\| \text { for all } n \in \mathbb{N} .
$$

Since $\sum_{n=1}^{\infty}\left\|y_{n}-T y_{n}\right\|^{2}<\infty$, it follows that $\sum_{n=1}^{\infty}\left\|x_{n}-T x_{n}\right\|^{2}<\infty$. Noticing that $R_{T}(n):=$ $R_{T,\left\{x_{n}\right\}}(n) \leq\left\|x_{n}-T x_{n}\right\|$ for all $n \in \mathbb{N}$ and that $\left\{R_{T}(n)\right\}$ is decreasing. Hence $\sum_{n=1}^{\infty}\left(R_{T}(n)\right)^{2}<\infty$. Therefore, from Lemma 2.3, we conclude that (21) holds.

\section{Appendix-IV: Proof of Proposition 4.2}

Proof Let $\left\{x_{n}\right\}$ be a sequence in $X$ such that $x_{n} \rightarrow z$ and $\left(I-S_{\lambda}\right) x_{n} \rightarrow 0$ as $n \rightarrow \infty$. We now show that $\left(I-S_{\lambda}\right) z=0$. If $F z=0$, then $z \in \Omega[\operatorname{VI}(C, F)]$, i.e, $\left(I-S_{\lambda}\right) z=0$. Assume that $F z \neq 0$. Let $x \in C$. Set $y_{n}=S_{\lambda} x_{n}$. From Lemma 2.1(a), we have

$$
\begin{aligned}
\left\langle x_{n}-\lambda F x_{n}-y_{n}, x-y_{n}\right\rangle & =\left\langle x_{n}-\lambda F x_{n}-P_{C}(I-\lambda F) x_{n}, x-P_{C}(I-\lambda F) x_{n}\right\rangle \\
& \leq 0 \text { for all } n \in \mathbb{N} .
\end{aligned}
$$

Hence

$$
\left\langle x_{n}-y_{n}, x-y_{n}\right\rangle \leq \lambda\left\langle F x_{n}, x-y_{n}\right\rangle
$$

which implies that

$$
\left\langle x_{n}-y_{n}, x-y_{n}\right\rangle \leq \lambda\left\langle F x_{n}, x-x_{n}+x_{n}-y_{n}\right\rangle
$$

i.e,

$$
\left\langle x_{n}-y_{n}, x-y_{n}\right\rangle-\lambda\left\langle F x_{n}, x_{n}-y_{n}\right\rangle \leq \lambda\left\langle F x_{n}, x-x_{n}\right\rangle .
$$

Since $x_{n} \rightarrow z,\left\|x_{n}-y_{n}\right\| \rightarrow 0$ and $F$ is sequentially weak-to-weak continuous, we have $y_{n} \rightarrow z$ and $F x_{n} \rightarrow F z$. Hence, from (65), we have

$$
0 \leq \liminf _{n \rightarrow \infty}\left\langle F y_{n}, x-y_{n}\right\rangle
$$

Since the norm is sequentially weakly lower semicontinuous, we get

$$
0<\|F z\| \leq \liminf _{n \rightarrow \infty}\left\|F y_{n}\right\|
$$

It follows that there exists $n_{0} \in \mathbb{N}$ such that $F y_{n} \neq 0$ for all $n \geq n_{0}$. Now we choose a sequence $\left\{\varepsilon_{k}\right\}$ in $(0, \infty)$ such that $\left\{\varepsilon_{k}\right\}_{k \geq 0}$ is strictly decreasing and $\varepsilon_{k} \rightarrow 0$. For $\left\{\varepsilon_{k}\right\}_{k \geq 0}$, from (66) and (67), there exists a strictly increasing sequence $\left\{n_{k}\right\}_{k \geq 0}$ of positive integers such that

$$
\left\langle F y_{n_{k}}, x-y_{n_{k}}\right\rangle+\varepsilon_{k} \geq 0 \text { and } F y_{n_{k}} \neq 0 \text { for all } k \geq 0 \text {. }
$$


Set $p_{k}=\frac{1}{\left\|F y_{n_{k}}\right\|^{2}} F y_{n_{k}}$. Then $\left\langle F y_{n_{k}}, p_{k}\right\rangle=1$. From (68), we gave

$$
\left\langle F y_{n_{k}}, x-y_{n_{k}}+\varepsilon_{k} p_{k}\right\rangle=\left\langle F y_{n_{k}}, x-y_{n_{k}}\right\rangle+\varepsilon_{k}\left\langle F y_{n_{k}}, p_{k}\right\rangle \geq 0 \text { for all } k \geq 0 \text {. }
$$

By the pseudo-monotonicity of $F$ on $X$, we have

$$
\left\langle F\left(x+\varepsilon_{k} p_{k}\right), x-y_{n_{k}}+\varepsilon_{k} p_{k}\right\rangle \geq 0 \text { for all } k \geq 0 .
$$

Note $F$ satisfies the condition $(\mathscr{B})$. Then, by the boundedness of $\left\{F y_{n_{k}}\right\}$, there exist constants $m, M>0$ such that $m \leq\left\|F y_{n_{k}}\right\| \leq M$ for all $k \geq 0$. Hence $\left\|\varepsilon_{k} p_{k}\right\|=\varepsilon_{k}\left\|p_{k}\right\|=\frac{\varepsilon_{k}}{\left\|F y_{n_{k}}\right\|} \rightarrow 0$ as $k \rightarrow \infty$. Thus, from (69), we get $\langle F x, x-z\rangle \geq 0$. Since $x$ is an arbitrary element in $C$, we infer that $z \in \Omega[\operatorname{DVI}(C, F)]$. Therefore, from Lemma 4.2, we conclude that $z \in \Omega[\operatorname{VI}(C, F)]$, i.e., $\left(I-S_{\lambda}\right) z=0$.

\section{Appendix-V: Proof of Proposition 4.3}

Proof (a) Let $u, v \in X$. Then, from Remark 4.1, we have

$$
\begin{aligned}
\left\|E_{\lambda}(u)-E_{\lambda}(v)\right\| & \leq \| u-v-\lambda\left(F S_{\lambda}(u)-F S_{\lambda}(v) \|\right. \\
& \leq\|u-v\|+\lambda L\left\|S_{\lambda}(u)-S_{\lambda}(v)\right\| \\
& \leq\left(1+\lambda L+\lambda^{2} L^{2}\right)\|u-v\| .
\end{aligned}
$$

(b) Let $x \in X$ and $v \in \Omega[\operatorname{VI}(C, F)]$. Set $y=P_{C}(x-\lambda F x)$ and $z=P_{C}(x-\lambda F y)$. Then $\langle F v, y-v\rangle \geq 0$. By the pseudo-monotonicity of $F$, we have $\langle F y, y-v\rangle \geq 0$. From Lemma 2.1(d), we have

$$
\begin{aligned}
\left\|P_{C}(x-\lambda F y)-v\right\|^{2} & \leq\|x-\lambda F y-v\|^{2}-\left\|x-\lambda F y-P_{C}(x-\lambda F y)\right\|^{2} \\
& =\|x-v-\lambda F y\|^{2}-\|x-z-\lambda F y\|^{2} \\
& =\|x-v\|^{2}-2 \lambda\langle F y, x-v\rangle+\lambda^{2}\|F y\|^{2}-\|x-z\|^{2}+2 \lambda\langle F y, x-z\rangle-\lambda^{2}\|F y\|^{2} \\
& =\|x-v\|^{2}-\|x-z\|^{2}+2 \lambda\langle F y, v-z\rangle \\
& =\|x-v\|^{2}-\|x-z\|^{2}+2 \lambda[\langle F y, v-y\rangle+\langle F y, y-z\rangle] \\
& \leq\|x-v\|^{2}-\|x-z\|^{2}+2 \lambda\langle F y, y-z\rangle .
\end{aligned}
$$

Substituting $x$ by $x-\lambda F x$ and $y$ by $z$ in Lemma 2.1(a), we get

$$
\left\langle x-\lambda F x-P_{C}(x-\lambda F x), z-P_{C}(x-\lambda F x)\right\rangle \leq 0,
$$

which gives that

$$
\langle x-\lambda F x-y, z-y\rangle \leq 0 .
$$

Thus, from (71), we have

$$
\begin{aligned}
\langle x-\lambda F y-y, z-y\rangle & =\langle x-\lambda F x-y, z-y\rangle+\langle\lambda F x-\lambda F y, z-y\rangle \\
& \leq \lambda\langle F x-F y, z-y\rangle \\
& \leq \lambda L\|x-y\|\|z-y\| \\
& \leq \frac{\lambda L}{2}\left(\|x-y\|^{2}+\|y-z\|^{2}\right) .
\end{aligned}
$$

Hence, from (70) and (72), we get

$$
\begin{aligned}
\|z-v\|^{2} & \leq\|x-v\|^{2}-\|x-y+(y-z)\|^{2}+2 \lambda\langle F y, y-z\rangle \\
& =\|x-v\|^{2}-\|x-y\|^{2}-\|y-z\|^{2}-2\langle x-y, y-z\rangle+2 \lambda\langle F y, y-z\rangle \\
& =\|x-v\|^{2}-\|x-y\|^{2}-\|y-z\|^{2}-2\langle x-\lambda F y-y, y-z\rangle \\
& \leq\|x-v\|^{2}-\|x-y\|^{2}-\|y-z\|^{2}+\lambda L\left(\|x-y\|^{2}+\|y-z\|^{2}\right) \\
& =\|x-v\|^{2}-(1-\lambda L)\|x-y\|^{2}-(1-\lambda L)\|y-z\|^{2} .
\end{aligned}
$$


For $\rho=1 / 2$, from (9), we have

$$
\|y-z\|^{2}=\|y-x+x-z\|^{2} \geq \frac{1}{2}\|x-z\|^{2}-\|y-x\|^{2} .
$$

Hence, from (73), we have

$$
\begin{aligned}
\|z-v\|^{2} & \leq\|x-v\|^{2}-(1-\lambda L)\|x-y\|^{2}-\frac{1-\lambda L}{2}\|x-z\|^{2}+(1-\lambda L)\|y-x\|^{2} \\
& =\|x-v\|^{2}-\frac{1-\lambda L}{2}\|x-z\|^{2} .
\end{aligned}
$$

(c) Lemma 4.1 shows that $\operatorname{Fix}\left(S_{\lambda}\right)=\Omega[\operatorname{VI}(C, F)]$. Note $\emptyset \neq \operatorname{Fix}\left(S_{\lambda}\right) \subseteq \operatorname{Fix}\left(E_{\lambda}\right)$. We now show that $\operatorname{Fix}\left(E_{\lambda}\right) \subseteq \operatorname{Fix}\left(S_{\lambda}\right)$. Let $u \in \operatorname{Fix}\left(E_{\lambda}\right)$ and $v \in \operatorname{Fix}\left(S_{\lambda}\right)$. From Part $\mathrm{b}(\mathrm{i})$, we get

$$
\begin{aligned}
\|u-v\|^{2}=\left\|E_{\lambda}(u)-v\right\|^{2} & \leq\|u-v\|^{2}-(1-\lambda L)\left\|u-S_{\lambda}(u)\right\|^{2}-(1-\lambda L)\left\|S_{\lambda}(u)-E_{\lambda}(u)\right\|^{2} \\
& =\|u-v\|^{2}-2(1-\lambda L)\left\|u-S_{\lambda}(u)\right\|^{2},
\end{aligned}
$$

which implies that $u \in \operatorname{Fix}\left(S_{\lambda}\right)$.

(d) Let $\lambda \in(0,1 / L)$. Let $\left\{x_{n}\right\}$ be a bounded sequence in $X$ such that $\lim _{n \rightarrow \infty}\left\|x_{n}-E_{\lambda} x_{n}\right\|=0$. Let $v \in \Omega[\operatorname{VI}(C, F)]$. From Part (b)(i), we have

$$
\left\|E_{\lambda}\left(x_{n}\right)-v\right\|^{2} \leq\left\|x_{n}-v\right\|^{2}-(1-\lambda L)\left\|x_{n}-S_{\lambda}\left(x_{n}\right)\right\|^{2} \text { for all } n \in \mathbb{N},
$$

it follows that

$$
\begin{aligned}
(1-\lambda L)\left\|x_{n}-S_{\lambda}\left(x_{n}\right)\right\|^{2} & \leq\left\|x_{n}-v\right\|^{2}-\left\|E_{\lambda}\left(x_{n}\right)-v\right\|^{2} \\
& =\left(\left\|x_{n}-v\right\|-\left\|E_{\lambda}\left(x_{n}\right)-v\right\|\right)\left(\left\|x_{n}-v\right\|+\left\|E_{\lambda}\left(x_{n}\right)-v\right\|\right) \\
& \leq 2\left\|x_{n}-v\right\|\left\|x_{n}-E_{\lambda}\left(x_{n}\right)\right\| \text { for all } n \in \mathbb{N} .
\end{aligned}
$$

By the boundedness of $\left\{x_{n}\right\}$, we have $\lim _{n \rightarrow \infty}\left\|x_{n}-S_{\lambda} x_{n}\right\|=0$.

\section{Appendix-VI: Proof of Proposition 4.4}

Proof (a) Let $u, v \in X$. Then

$$
\left\|T_{\lambda} u-T_{\lambda} v\right\| \leq\left\|S_{\lambda} u-S_{\lambda} v\right\|+\lambda\left\|F S_{\lambda} u-F S_{\lambda} v\right\|+\lambda\|F u-F v\| \leq\left(1+3 \lambda L+\lambda^{2} L^{2}\right)\|u-v\| .
$$

(b) Let $\lambda \in(0,1 / L), x \in X$ and $z \in \Omega[\operatorname{VI}(C, F)]$. Set $y:=P_{C}(x-\lambda F x)$. Hence

$$
\left\|T_{\lambda} x-x\right\|=\|y-x-\lambda(F y-F x)\| \leq\|y-x\|+\lambda\|F y-F x\| \leq(1+\lambda L)\|y-x\|
$$

and

$$
\left\|S_{\lambda} x-x\right\|=\left\|T_{\lambda} x-x+\lambda(F y-F x)\right\| \leq\left\|T_{\lambda} x-x\right\|+\lambda L\|y-x\|,
$$

which implies that $(1-\lambda L)\left\|x-S_{\lambda} x\right\| \leq\left\|x-T_{\lambda} x\right\|$. From Lemma 2.1(a), we have

$$
\begin{aligned}
y=P_{C}(x-\lambda F x) & \Leftrightarrow\langle y-x+\lambda F x, y-z\rangle \leq 0 \\
& \Leftrightarrow\langle y-x, y-z\rangle \leq-\lambda\langle F x, y-z\rangle .
\end{aligned}
$$


Thus,

$$
\begin{aligned}
\left\|T_{\lambda} x-z\right\|^{2}= & \|y-\lambda(F y-F x)-z\|^{2} \\
= & \|y-z\|^{2}+\lambda^{2}\|F y-F x\|^{2}-2 \lambda\langle F y-F x, y-z\rangle \\
= & \|y-x+x-z\|^{2}+\lambda^{2}\|F y-F x\|^{2}-2 \lambda\langle F y-F x, y-z\rangle \\
= & \|y-x\|^{2}+\|x-z\|^{2}+2\langle y-x, x-z\rangle+\lambda^{2}\|F y-F x\|^{2}-2 \lambda\langle F y-F x, y-z\rangle \\
= & \|y-x\|^{2}+\|x-z\|^{2}+2\langle y-x, x-y\rangle+2\langle y-x, y-z\rangle \\
& +\lambda^{2}\|F y-F x\|^{2}-2 \lambda\langle F y-F x, y-z\rangle \\
= & \|x-z\|^{2}-\|y-x\|^{2}+2\langle y-x, y-z\rangle+\lambda^{2}\|F y-F x\|^{2}-2 \lambda\langle F y-F x, y-z\rangle \\
\leq & \|x-z\|^{2}-\|y-x\|^{2}+\lambda^{2}\|F y-F x\|^{2}-2 \lambda\langle F x, y-z\rangle-2 \lambda\langle F y-F x, y-z\rangle \\
\leq & \|x-z\|^{2}-\|y-x\|^{2}+\lambda^{2} L^{2}\|y-x\|^{2}-2 \lambda\langle F y, y-z\rangle .
\end{aligned}
$$

Since $z \in \Omega[\operatorname{VI}(C, F)]$, we have $\langle F z, y-z\rangle \geq 0$. By pseudo-monotonicity of $F$, we have $\langle F y, y-z\rangle \geq$ 0. From (74), we have

$$
\left\|T_{\lambda} x-z\right\|^{2} \leq\|x-z\|^{2}-\left(1-\lambda^{2} L^{2}\right)\left\|x-S_{\lambda} x\right\|^{2}
$$

and hence

$$
\left\|T_{\lambda} x-z\right\|^{2} \leq\|x-z\|^{2}-\kappa\left\|x-T_{\lambda} x\right\|^{2} .
$$

(c) Note $\operatorname{Fix}\left(S_{\lambda}\right)=\Omega[\operatorname{VI}(C, F)]$. Observe that $\operatorname{Fix}\left(S_{\lambda}\right) \subseteq \operatorname{Fix}\left(T_{\lambda}\right)$. We now show that $\operatorname{Fix}\left(T_{\lambda}\right) \subseteq$ $\operatorname{Fix}\left(S_{\lambda}\right)$. Let $u \in \operatorname{Fix}\left(T_{\lambda}\right)$ and $v \in \operatorname{Fix}\left(S_{\lambda}\right)$. From Part $\mathrm{b}(\mathrm{iii})$, we get

$$
\|u-v\|^{2}=\left\|T_{\lambda}(u)-v\right\|^{2} \leq\|u-v\|^{2}-\left(1-\lambda^{2} L^{2}\right)\left\|u-S_{\lambda}(u)\right\|^{2},
$$

which implies that $u \in \operatorname{Fix}\left(S_{\lambda}\right)$.

(d) Let $\lambda \in(0,1 / L)$. Let $\left\{x_{n}\right\}$ be a bounded sequence in $X$ such that $\lim _{n \rightarrow \infty}\left\|x_{n}-T_{\lambda} x_{n}\right\|=0$. Using Part (b)(ii), we obtain

$$
(1-\lambda L)\left\|x_{n}-S_{\lambda} x_{n}\right\| \leq\left\|x_{n}-T_{\lambda} x_{n}\right\| \text { for all } n \in \mathbb{N} .
$$

Thus, we conclude that the operator $S_{\lambda}$ has property $(\mathscr{A})$ with respect to the operator $T_{\lambda}$.

\section{Appendix-VII: Proof of Example 5.2}

To prove the pseudo-monotonicity and Lipschitz continuity of operator $F$ defined by (46), we define $g: X \rightarrow \mathbb{R}$ such that $g(x)=e^{-\langle x, U x\rangle}+\alpha$ for all $x \in X$. Let $x, y \in X$ such that $\langle F x, y-x\rangle \geq 0$. Since $g(x)>0$, it follows that $\langle V x+q, y-x\rangle \geq 0$. Hence

$$
\begin{aligned}
\langle F y, y-x\rangle=g(y)\langle V y+q, y-x\rangle & \geq g(y)(\langle V y+q, y-x\rangle-\langle V x+q, y-x\rangle) \\
& =g(y)\langle V(y-x), y-x\rangle \\
& \geq 0 .
\end{aligned}
$$

Thus, $F$ is pseudo-monotonous.

Now, let $x, h \in X$. Then $\nabla F(x)(h)=2 e^{-\langle x, U x\rangle}\langle U x, h\rangle(V x+p)+\left(e^{-\langle x, U x\rangle}+\alpha\right) V h$ and

$$
\|\nabla F(x)(h)\| \leq 2 e^{-\eta\|x\|^{2}}\|U\|\|x\|(\|V\|\|x\|+\|p\|)\|h\|+\left(e^{-\eta\|x\|^{2}}+\alpha\right)\|V\|\|h\| .
$$

Hence

$$
\|\nabla F(x)\| \leq 2 e^{-\eta\|x\|^{2}}\|U\|\|x\|(\|V\|\|x\|+\|p\|)+\left(e^{-\eta\|x\|^{2}}+\alpha\right)\|V\| .
$$




\section{Compliance with ethical standards}

Conflict of interest The author declares that there is no conflict of interests regarding the publication of this paper.

Ethical approval This article does not contain any studies with human participants or animals performed by the author.

\section{References}

1. R. P. Agarwal, D. O'Regan, and D. R. Sahu. Fixed Point Theory for Lipschitzian-type Mappings with Applications. Springer-Verlag New York, 1st edition, 2009.

2. R. P. Agarwal, Donal O Regan, and DR Sahu. Iterative construction of fixed points of nearly asymptotically nonexpansive mappings. Journal of Nonlinear and convex Analysis, 8(1):61, 2007.

3. F. Alvarez. Weak convergence of a relaxed and inertial hybrid projection-proximal point algorithm for maximal monotone operators in Hilbert space. SIAM Journal on Optimization, 14(3):773-782, 2004.

4. F. A. and H. Attouch. An inertial proximal method for maximal monotone operators via discretization of a nonlinear oscillator with damping. Set-Valued Analysis, 9(1-2):3-11, 2001.

5. Q. H. Ansari and D. R. Sahu. Some iterative methods for fixed point problems. In Topics in Fixed Point Theory, pages 273-300. Springer, 2014.

6. Q. H. Ansari and D. R.Sahu. Extragradient methods for some nonlinear problems. In Fixed Point Theory and Graph Theory, pages 187-230. Elsevier, 2016.

7. H. H. Bauschke and P. L. Combettes. Convex analysis and monotone operator theory in Hilbert spaces, volume 408. Springer, 2011.

8. R. I. Bot, E. R. Csetnek, and C. Hendrich. Inertial Douglas-Rachford splitting for monotone inclusion problems. Applied Mathematics and Computation, 256:472-487, 2015.

9. R.I. Bot, E. R. Csetnek, and P. T. Vuong. The forward-backward-forward method from continuous and discrete perspective for pseudo-monotone variational inequalities in Hilbert spaces. European Journal of Operational Research, 287(1):49-60, 2020.

10. Y. Censor, A. Gibali, and S. Reich. The subgradient extragradient method for solving variational inequalities in Hilbert space. Journal of Optimization Theory and Applications, 148(2):318-335, 2011.

11. C. Chidume. Geometric properties of Banach spaces and nonlinear iterations, volume 1965. Springer, 2009.

12. A. Dixit, D. R. Sahu, A. K. Singh, and T. Som. Application of a new accelerated algorithm to regression problems. Soft Computing, pages 1-14, 2019.

13. Q. L. Dong, J. Huang, X. H. Li, Y. J.Cho, and T. M. Rassias. Mikm: multi-step inertial Krasnoselskii-Mann algorithm and its applications. Journal of Global Optimization, 73(4):801-824, 2019.

14. Q. L. Dong, Y. Y. Lu, and J. Yang. The extragradient algorithm with inertial effects for solving the variational inequality. Optimization, 65(12):2217-2226, 2016.

15. Y. Dong. Comments on "the proximal point algorithm revisited". Journal of Optimization Theory and Applications, 166(1):343-349, 2015.

16. A. A., Goldstein. Convex programming in Hilbert space. Bulletin of the American Mathematical Society, 70(5):709-710, 1964.

17. C. Kanzow and Y. Shehu. Generalized Krasnoselskii-Mann-type iterations for nonexpansive mappings in hilbert spaces. Computational Optimization and Applications, 67(3):595-620, 2017.

18. T. Kato. Demicontinuity, hemicontinuity and monotonicity. Bulletin of the American Mathematical Society, 70(4):548-550, 1964.

19. P. D. Khanh. A modified extragradient method for infinite-dimensional variational inequalities. Acta Mathematica Vietnamica, 41(2):251-263, 2016.

20. G. M. Korpelevich. The extragradient method for finding saddle points and other problems. Matecon, 12:747756, 1976.

21. P. K. Anh, D. V. Thong, and N. T. Vinh. Improved inertial extragradient methods for solving pseudo-monotone variational inequalities. Optimization, pages 1-24, 2020.

22. P. E. Maingé. Convergence theorems for inertial KM-type algorithms. Journal of Computational and Applied Mathematics, 219(1):223-236, 2008.

23. W. R. Mann. Mean value methods in iteration. Proceedings of the American Mathematical Society, 4(3):506510, 1953.

24. B. T. Polyak. Some methods of speeding up the convergence of iteration methods. USSR Computational Mathematics and Mathematical Physics, 4(5):1-17, 1964.

25. D. R. Sahu. Applications of the S-iteration process to constrained minimization problems and split feasibility problems. Fixed Point Theory, 12(1):187-204, 2011.

26. D. R. Sahu. Applications of accelerated computational methods for quasi-nonexpansive operators to optimization problems. Soft Computing, pages 1-25, 2020.

27. D. R. Sahu, Q. H. Ansari, and J. C. Yao. Convergence of inexact Mann iterations generated by nearly nonexpansive sequences and applications. Numerical Functional Analysis and Optimization, 37(10):1312-1338, 2016.

28. D. R. Sahu, A. Pitea, and M. Verma. A new iteration technique for nonlinear operators as concerns convex programming and feasibility problems. Numerical Algorithms, https://doi.org/10.1007/s11075-019-00688-9, 2019 . 
29. D. R. Sahu, L. Shi, N. C. Wong, and J. C. Yao. Perturbed iterative methods for a general family of operators: convergence theory and applications. Optimization, pages 1-37, 2020.

30. D. R. Sahu, N. C. Wong, and J. C. Yao. A unified hybrid iterative method for solving variational inequalities involving generalized pseudocontractive mappings. SIAM Journal on Control and Optimization, 50(4):2335$2354,2012$.

31. D. R. Sahu, J. C. Yao, M. Verma, and K. K. Shukla. Convergence rate analysis of proximal gradient methods with applications to composite minimization problems. Optimization, pages 1-26, 2020.

32. D. R. Sahu, J. C. Yao, V. K. Singh, and S. Kumar. Semilocal convergence analysis of S-iteration process of Newton-Kantorovich like in Banach spaces. Journal of Optimization Theory and Applications, 172(1):102-127, 2017.

33. D. R. Sahu and A. K. Singh. Inertial iterative algorithms for common solution of variational inequality and system of variational inequalities problems. Journal of Applied Mathematics and Computing, 65(1):351-378, 2021.

34. D.V. Thong and D, V, Hieu. Modified Tseng's extragradient algorithms for variational inequality problems. Journal of Fixed Point Theory and Applications, 20(4):1-18, 2018.

35. P. Tseng. A modified forward-backward splitting method for maximal monotone mappings. SIAM Journal on Control and Optimization, 38(2):431-446, 2000.

36. M. Verma, D. R. Sahu, and K. K. Shukla. Vaga: a novel viscosity-based accelerated gradient algorithm. Applied Intelligence, pages 1-15, 2017.

37. P. T. Vuong. On the weak convergence of the extragradient method for solving pseudo-monotone variational inequalities. Journal of optimization theory and applications, 176(2):399-409, 2018.

38. H. K. Xu. Iterative algorithms for nonlinear operators. Journal of the London Mathematical Society, 66(1):240$256,2002$.

39. Y. Yao, G. Marino, and L. Muglia. A modified korpelevich's method convergent to the minimum-norm solution of a variational inequality. Optimization, 63(4):559-569, 2014.

40. E. Zeidler. Nonlinear Functional Analysis and Its Applications: III: Variational Methods and Optimization. Springer Science \& Business Media, 2013.

41. Y. C. Zhang, K. Guo, and T. Wang. Generalized Krasnoselskii-Mann-type iteration for nonexpansive mappings in banach spaces. Journal of the Operations Research Society of China, pages 1-12, 2019. 